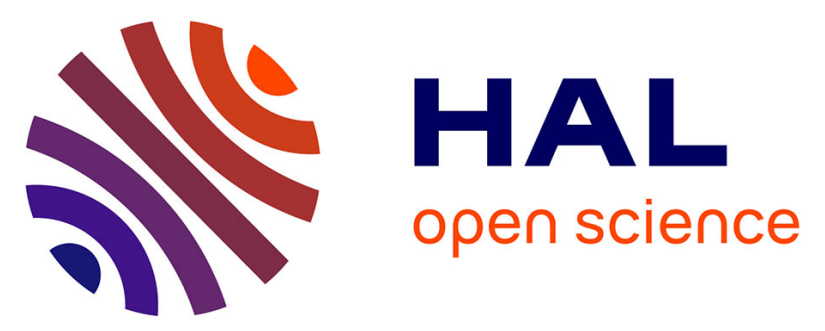

\title{
Comparison of different treatment schemes in 5-ALA interstitial photodynamic therapy for high-grade glioma in a preclinical model: An MRI study
}

Maximilien Vermandel, Mathilde Quidet, Anne-Sophie Vignion-Dewalle, Henri-Arthur Leroy, Bertrand Leroux, Serge Mordon, Nicolas Reyns

\section{To cite this version:}

Maximilien Vermandel, Mathilde Quidet, Anne-Sophie Vignion-Dewalle, Henri-Arthur Leroy, Bertrand Leroux, et al.. Comparison of different treatment schemes in 5-ALA interstitial photodynamic therapy for high-grade glioma in a preclinical model: An MRI study. Photodiagnosis and Photodynamic Therapy, 2018. hal-01975992

\section{HAL Id: hal-01975992 \\ https://hal.science/hal-01975992}

Submitted on 9 Jan 2019

HAL is a multi-disciplinary open access archive for the deposit and dissemination of scientific research documents, whether they are published or not. The documents may come from teaching and research institutions in France or abroad, or from public or private research centers.
L'archive ouverte pluridisciplinaire HAL, est destinée au dépôt et à la diffusion de documents scientifiques de niveau recherche, publiés ou non, émanant des établissements d'enseignement et de recherche français ou étrangers, des laboratoires publics ou privés. 


\section{Comparison of different treatment schemes in 5-ALA interstitial photodynamic therapy for high-grade glioma in a preclinical model: An MRI study}

Authors:

Maximilien Vermandel, $\mathrm{PhD}^{1,2}$, Mathilde Quidet, $\mathrm{MD}^{2}$, Anne-Sophie Vignion-Dewalle, $\mathrm{PhD}^{1}$, Henri-Arthur Leroy, $\mathrm{MD}^{1,2}$, Bertrand Leroux ${ }^{1}$, Serge Mordon, $\mathrm{PhD}^{1}$, Nicolas Reyns, $\mathrm{MD}, \mathrm{PhD}^{1,2}$

Affiliations:

${ }^{1}$ Univ. Lille, INSERM, CHU Lille, U1189 - ONCO-THAI - Image-Assisted Laser Therapy for Oncology, F-59000 Lille, France

${ }^{2}$ Department of Neurosurgery, University Hospital, F-59000 Lille, France

Corresponding author:

Dr. Maximilien Vermandel

INSERM U1189 - ONCO-THAI

University of Lille - CHRU de Lille

1, Avenue Oscar Lambret

Email: maximilien.vermandel@chru-lille.fr

Phone number: 0033320446721 


\section{Compliance with ethical standards:}

\section{Conflicts of interest: None.}

Funding: Financial support provided by the University of Lille, INSERM and the French Society of Neurosurgery.

Ethical approval: This article contains studies performed using animals. All applicable institutional and national guidelines for the care and use of animals were followed. The experimental design was declared to the French National Ethics Committee under reference number 04870.01 .

Authorship: M. Vermandel designed the study, cosupervised the study and wrote the

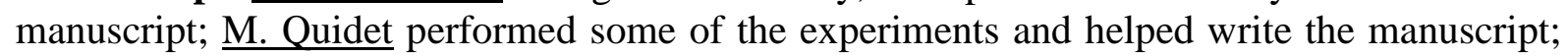
AS. Vignion-Dewalle performed the statistical analysis and helped write the manuscript; $\underline{H A}$ Leroy performed some of the experiments and read/corrected the manuscript; B. Leroux performed some of the experiments and reported results; and $\underline{\mathrm{S} \text {. Mordon and N. Reyns }}$ cosupervised the study, designed the study and read/corrected the manuscript.

Acknowledgements: The authors warmly thank Florent Auger and Nicolas Durieux from the University of Lille for their helpful support in acquiring the MR images. 


\section{Background}

There is currently no therapy that prevents high-grade glioma recurrence. Thus, these primary brain tumors have unfavorable outcomes. Recently, 5-ALA photodynamic therapy (PDT) has been proposed to delay relapse and is highly expected to have potential synergistic effects with the current standard of care. However, PDT treatment delivery needs to be optimized by evaluating the impact of both the number of fractions and the light power used.

\section{Objectives}

Previous studies have reported MRI examination-based outcomes for PDT in glioblastoma. Our study aimed to compare MRI markers across different treatment schemes that use interstitial PDT in high-grade glioma in a preclinical model.

\section{Materials and Methods}

Forty-eight "nude" rats were grafted with human U87 cells into the right putamen and subsequently submitted to interstitial PDT. The rats were randomized into six groups, including two different sham groups and four different treated groups ( 5 fractions at $5 \mathrm{~mW}$ or $30 \mathrm{~mW}$ and 2 fractions at $5 \mathrm{~mW}$ or $30 \mathrm{~mW}$ ). After photosensitizer (PS) precursor (5-ALA) intake, an optical fiber was introduced into the tumor. Treatment effects were assessed with early high-field MRI to acquire T1 and T2 diffusion and perfusion images.

\section{Results}

There was no difference in the variation of the diffusion coefficient among the six groups ( $\mathrm{p}=0.0549$, Kruskal-Wallis test). However, a significant difference was identified among the six groups in terms of variation in perfusion ( $\mathrm{p}=0.048$, Kruskal-Wallis test), supporting a lesional effect in the treated groups. Additionally, the sham groups had significantly smaller edema volumes than were observed in the treated groups. Moreover, the 5-fraction group treated with $30 \mathrm{~mW}$ was associated with edema volumes that were significantly greater than those in the 5-fraction group treated with $5 \mathrm{~mW}(\mathrm{p}=0.019)$.

\section{Conclusion}

Based on observations of MRI data and considering treatment effects, the 5-fraction group treated at $5 \mathrm{~mW}$ was not significantly different from the other treated groups in terms of cell 
deaths, characterized by diffusion imaging, or necrosis level. However, the significantly lower level of edema observed in this group indicated that this treatment scheme had limited toxicity.

Keywords: 5-ALA; PpIX, Photodynamic Therapy; Magnetic Resonance Imaging; PDT; MRI; Glioblastoma; High-Grade Glioma 


\section{Introduction}

Glioblastoma (GBM) is a malignant brain tumor with a particularly poor prognosis and a median overall survival of approximately 15 months given the current standard of care (SOC) (1-3). Although GBM is a rare neoplastic disease with a low prevalence $(0.3 / 10,000$ persons), it remains the most frequent primary malignant brain tumor in adults $(4,5)$.

Currently, the European Society of Medical Oncology (ESMO) guidelines provide recommended treatment options according to the grade of the glioma (6). Given the invasive nature of GBM, local relapse is a universal occurrence and is the reason that surgery is followed by radiotherapy (RT) to the tumor bed and concomitant and adjuvant temozolomide chemotherapy (TMZ), which prolong both progression-free survival (PFS) and overall survival (OS). Although adjuvant treatment after surgery significantly improves results, its benefits remain modest (less than 3 months over the median OS with RT+TMZ versus RT alone (3)). Therefore, the management of newly diagnosed patients should include maximal resection when achievable. The extent of resection (EOR) has prognostic value.

Currently, no existing therapeutic agent is available that can stop GBM progression, and complete resection is rarely feasible because tumor cells usually infiltrate the surrounding brain tissue. Adjuvant therapies to improve local control are thus highly expected.

Recently, 5 amino levulinic acid (5-ALA) photodynamic therapy (PDT) was proposed to be delivered intraoperatively during GBM resection within the INDYGO clinical trial ${ }^{1}$. Although this trial aims to investigate the safety and feasibility of 5-ALA PDT during neurosurgery procedures, it is highly expected to have potential synergistic effects with SOC in the patients enrolled in the trial who harbor newly diagnosed GBM.

The concept underlying 5-ALA PDT relies on the specific accumulation of the photosensitizer protoporphyrin IX (PpIX) in GBM cells because they lack several enzymatic cascades, which usually leads to heme biosynthesis. After PpIX is excited with a $635 \mathrm{~nm}$ laser light for 5-ALA PDT, free radical production and oxidative species formation (7) lead to cytotoxic reactions

\footnotetext{
${ }^{1}$ https://clinicaltrials.gov/ct2/show/NCT03048240
} 
within GBM cells. The presence of oxygen is thus required, and treatment delivery should involve tissue oxygenation to maximize therapeutic effects(8). Alternating "on" and "off" periods of light (i.e., fractionation) might improve tissue reoxygenation during the "off" periods that follow oxygen consumption during the "on" periods to address this issue (9).

In a previous study, we used 2-fraction and 5-fraction schemes to deliver the same light dose of $25 \mathrm{~J}$, and we demonstrated that light fractionation during interstitial PDT (iPDT) enhanced therapeutic effects $(10,11)$. These effects were assessed in a preclinical GBM model using both histopathological analyses and non-invasive techniques that relied on MRI imaging(12). 5-ALA PDT induced targeted tumor lesions, and its primary effects included necrosis, apoptosis, and reduced central tumor vascularization, as confirmed by immunohistology. We also observed that diffusion and perfusion MRI sequences accurately assess these treatment effects. Indeed, diffusion was a pertinent marker of cell death, and perfusion provided a good indication of the level of necrosis. Additionally, and as expected, T2 MRI was clearly confirmed to be a precise estimator of peritumoral edema.

In studies by Leroy et al.(10, 11), the major treatment effect of high-power iPDT was intratumoral necrosis associated with surrounding edema and a higher vascular density corresponding to an inflammatory response in the periphery of the treated tumor. Although the 5-fraction treatment was more effective in terms of tumor-induced necrosis, it was associated with more extensive post-treatment perilesional edema and was thus associated with higher toxicity. Indeed, apoptosis is the preferred cell death pathway for limiting inflammation and excess edema.

In this context, using a light power lower than that used in $(10,11)$ might enhance PDT efficiency by preserving tumor oxygenation $(13,14)$. Additionally, low light power has been suggested to induce selective apoptosis, which causes less damage than is caused by necrosis to normal adjacent brain tissues (15-17). Thus, to optimize tumor cytotoxicity while limiting edema and toxicity in adjacent healthy brain tissues, we studied the effects of low-power iPDT on the same murine glioblastoma model.

MRI examinations were performed to monitor the low-power iPDT response, and data were combined with previous MRI data acquired alongside the previous high-power iPDT study. This method made a comprehensive analysis of the different treatment schemes feasible. 


\section{Materials and methods}

The main objective of this study was to evaluate the effects of two distinct light delivery regimens delivered at two distinct light powers, leading to four distinct treatment schemes: 1) 2-fractions and 2) 5-fractions delivered at $30 \mathrm{~mW}$ each and 3) 2-fractions and 4) 5-fractions delivered at $5 \mathrm{~mW}$ each. The same doses of 5-ALA were prescribed, and the same treatment devices and the same light dose of $25 \mathrm{~J}$ were used. The resulting four treatment groups were compared to two sham groups: 1) with 5-ALA and without light and 2) without 5-ALA and with light ( 2 fractions at $5 \mathrm{~mW}$ ) to assess the effects of either the drug or the laser light alone.

a) Preclinical model

- Ethical considerations

All operative and animal care procedures were performed in line with the French Government's guidelines (decree 2001-464, 29 May 2001) and those of the Laboratory Animal Care and Use Committee. Our institutional ethics review board approved the study protocol ( $\mathrm{N}^{\circ}$ 01878.01).

- Murine model

All specimens were nude rats with a phenotype of Fox $\mathrm{n} 1 \mathrm{rnu} / \mathrm{rnu}$ and were obtained from Charles River ${ }^{\circledR}$ (Wilmington, MA, USA) and Envigo ${ }^{\circledR}$ (former Harlan ${ }^{\circledR}$, Gannat, France). These nude immune-compromised rats better tolerate experimental xenografts and have no high risk of post-operative infection(18). A quarantine period of 10 days from reception was observed before performing GBM cell grafting. Grafted rats were 8 weeks old and weighed approximately $150 \mathrm{~g}$.

- Grafting

To xenograft the GBM tumors, human GBM cells from the cell line U87 were used. Cell cultures as well as surgical grafting procedures were strictly identical to those described in previous studies(10, 12, 19). Surgical grafting was performed under local and general anesthesia administered via a mask delivering isoflurane. A stereotactic frame allowed a micromanipulator to be moved across the 3 planes of space with a $0.01 \mathrm{~mm}$ precision. The skull was immobilized horizontally via a tooth bar and two ear bars. A burr hole was drilled at the coordinates mentioned in the Paxinos and Watson atlas $(2.70 \mathrm{~mm}$ right and $0.50 \mathrm{~mm}$ 
anterior to bregma) to achieve surgical grafting in the right putamen. In all, $5 \times 10^{5}$ U87 glioblastoma cells were injected via a microsyringe connected to an automatic syringe pump.

A cranial anchor was used to guide the needle of the microsyringe and subsequently the optical fiber used for laser light delivery. This anchor was positioned in the burr hole and glued with biological adhesive to the outside of the skull.

b) Treatment

Treatments were delivered 12 days after the xenografts were performed. Animals randomly assigned to one of the treatment groups or to the sham group treated with 5-ALA received an intraperitoneal injection of 5-ALA (100 mg/kg) 4 hours prior to light excitation(20). Their cages were then covered with a light-occlusive dressing for 24 hours to prevent skin photosensitization (such as erythema or a burn). Then, all animals, regardless of group, were anaesthetized as previously described and placed on the MRI table. A bare flat-end silica fiber UltraSil 272 ULS (OFS, Norcross, USA) with an outside diameter of $350 \mu \mathrm{m}$ and a numerical aperture of 0.29 was guided into the tumor through the anchor set up during the grafting procedure. T1-weighted MRI was performed to confirm the optimal positioning of the fiber.

Depending on the randomized group, light was delivered interstitially via a $635 \mathrm{~nm}$ laser diode. All animals in the treatment groups or the sham group treated with light were subjected to a total dose of $25 \mathrm{~J}$ at an irradiance of $30 \mathrm{~mW}$ (Figure 1a) or $5 \mathrm{~mW}$ (Figure 1b) using variant fractionation schemes that depended on the treatment group (Figure 1). The fractionation regimen used in the 2-fraction group was previously documented by Curnow et al. (8). For this fractionation regimen, the light was stopped after $5 \mathrm{~J}$ before the remaining $20 \mathrm{~J}$ were delivered. This regimen was reported to produce an area of necrosis more than three times larger than that produced with continuous illumination (8). 
2-fractions@30mW

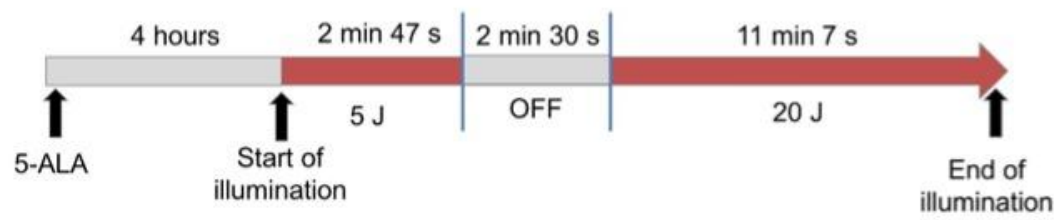

5-fractions@30mW

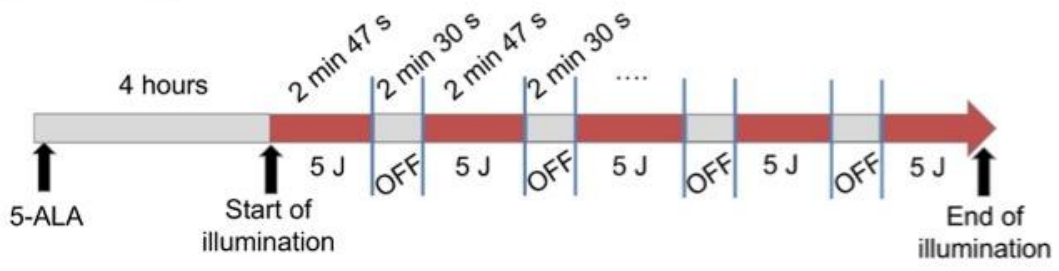

(a)

2-fractions@5mW

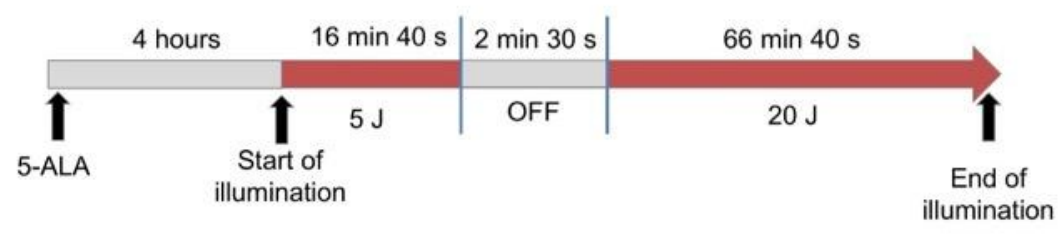

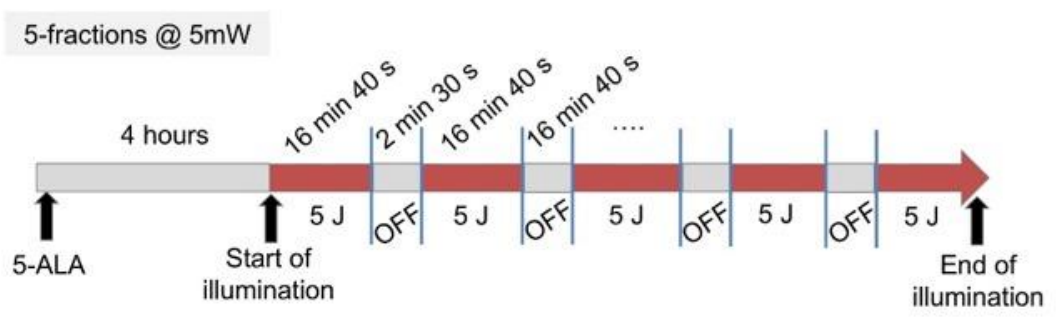

(b)

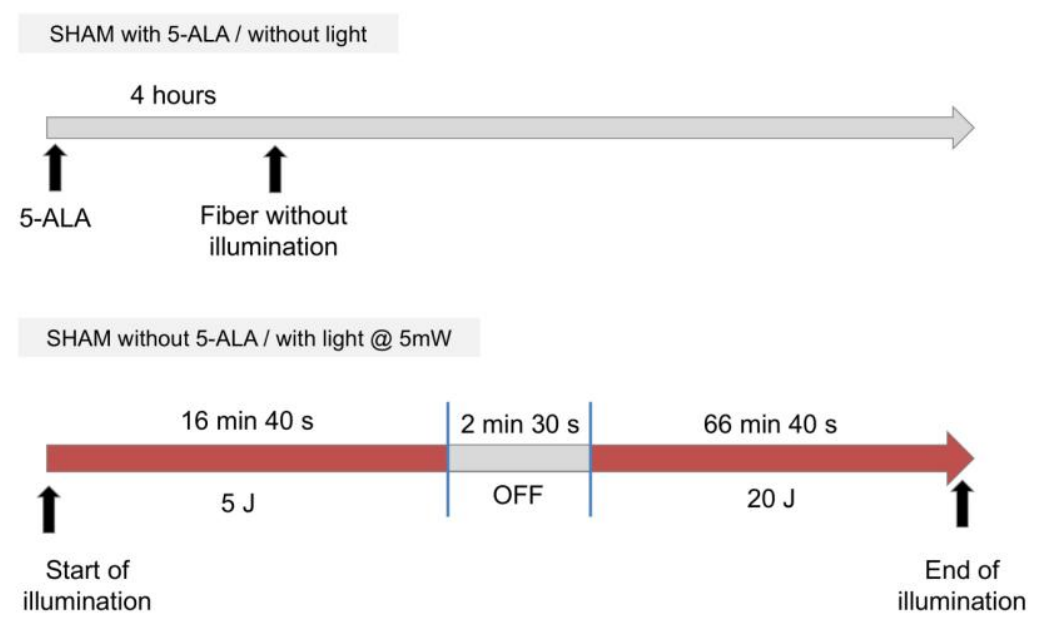

(c)

Figure 1: Description of irradiation schemes used in each group (sham, 2 fractions, or 5 
fractions). The total dose of delivered energy was $25 \mathrm{~J}$ at a power of $30 \mathrm{~mW}$ or $5 \mathrm{~mW}$ (measured at the fiber tip). The following groups are shown: (a) 2 or 5 fractions delivered at a power of $30 \mathrm{~mW}$, (b) 2 or 5 fractions delivered at a power of $5 \mathrm{~mW}$, (c) sham groups treated with 5-ALA/without light to check the hypothetical effect of the drug itself or without 5-ALA/with light to check the hypothetical effect of the laser light itself.

At the end of the procedure, the animals received $3 \mathrm{mg}$ of intraperitoneal methylprednisolone immediately after PDT to limit treatment-induced edema.

c) Imaging protocol and analysis

A 7 Tesla MRI was used to perform imaging (Bruker, Biospec, Ettlingen, Germany). Seven days after xenografting, tumor growth was assessed using a T2-weighted TurboRARE sequence(21) to observe the quality and extent of engraftment. Twelve days after xenografting, the animals received a pretreatment MRI immediately before undergoing iPDT. Fiber insertion was thus achieved when the tumor graft, as indicated on the MRI, had achieved a minimum coronal diameter greater than $2 \mathrm{~mm}$, and post-treatment MRI was acquired 48-72 hours later.

For follow-up purposes and treatment evaluation, pre- and post-treatment imaging systematically included T2 and T1 perfusion- and diffusion-weighted sequences. T2-weighted TurboRARE sequences(21) were acquired in the axial plane to evaluate the geometry and position of the tumor. A T1-weighted sequence was performed before and after gadolinium injection on the axial plane using the RARE type of spin echo. The perfusion sequence was performed using the arterial spin labeling (ASL)(22) method. This method provided quantitative information on tissue perfusion by magnetically labeling the blood entering the slice. This corresponded to the wash-in measurement. Diffusion-weighted imaging was achieved in 3D using the Echo-planar Imaging (EPI) technique, which provides information on the degrees of freedom of the water molecules in the tissue.

Post-processing of MRI data for diffusion and perfusion images was performed using Paravision 5.1 software provided by the manufacturer (Bruker@), Biospec, Ettlingen, Germany). Thus, tissue perfusion and diffusion coefficients were measured on axial slices with a thickness of $850 \mu \mathrm{m}$ and are expressed in units of $\mathrm{ml} /(100 \mathrm{~g}$ tissue $/ \mathrm{min})$ and $\mu \mathrm{m}^{2} / \mathrm{sec}$, respectively. Then, semiquantitative measurements of diffusion and perfusion values were computed from regions of interest (ROIs) placed 1) on the tumor and 2) contralateral to the 
tumor (striatum). The ratio between these two ROIs (tumor/healthy brain) was calculated preand post-treatment to calculate the delta (i.e., $\Delta \mathrm{d}$ for diffusion and $\Delta \mathrm{p}$ for perfusion) (Eq. 1).

$$
\Delta x=\frac{\text { ratio }_{\text {post }}-\text { ratio }_{\text {pre }}}{\text { ratio }_{\text {pre }}} \times 100 \%
$$

$x$ is the coefficient being observed according to the MRI sequence (i.e., diffusion or perfusion)

ratio $_{\text {pre }}=\frac{x_{\text {tumor }} \text { pre }}{x_{\text {striatumpre }}}$ and ratio $_{\text {post }}=\frac{x_{\text {tumor }_{\text {post }}}}{x_{\text {striatum }} \text { post }}$

and $x_{\text {tumor_pre }}, x_{\text {striatum_pre }}, x_{\text {tumor_post }}$ and $x_{\text {striatum_post }}$ indicates the average coefficient $x$ (i.e., diffusion or perfusion) measured for Tumor_ROI, Striatum_ROI pre- and post- treatment, respectively.

The tumor, necrosis and edema volumes were measured after manual delineation of the T1 and T2 MRI slices (OsiriX 6.5.2, Pixmeo, Switzerland). In particular, tumors were manually delineated via T1 gadolinium MRI, peritumoral edema was delineated on T2 images, and changes in intratumoral signals were manually delineated on either T2 or T1 gadolinium sequences to quantify necrosis.

d) Statistical analysis

Due to the small sample sizes (between 5 and 11 per group), non-parametric tests were used in this study. First, a Kruskal-Wallis test was conducted to evaluate differences in pretreatment tumor volumes among the six groups. If no significant differences were found, a further statistical analysis was performed. This analysis consisted of comparing the delta diffusion, delta perfusion, edema volume and necrosis volume among the six groups using the Kruskal-Wallis test. In cases with significant differences, multiple pairwise comparisons between the corresponding groups were performed using the Dwass-Steel-Critchlow-Fligner post hoc test. Data were analyzed with XLSTAT software 2013 for Windows (Addinsoft, New-York, NY). All significance levels were set to $5 \%$. 


\section{Results}

Forty-eight specimens harbored tumors that were sufficiently large for treatment and underwent post-MRI examination. The median tumor volume, measured from T1 MRI, was $4.00 \mathrm{~mm}^{3}$ (range, 1.04 to 19.01). The Kruskal-Wallis test showed there were no differences in pre-treatment tumor volumes between the six groups $(\mathrm{p}=0.503)$, making further analysis possible.

Figures 2, 3, 4, 5 and 6 show illustrations of different groups compared before and after treatment.

Pre-treatment MRI

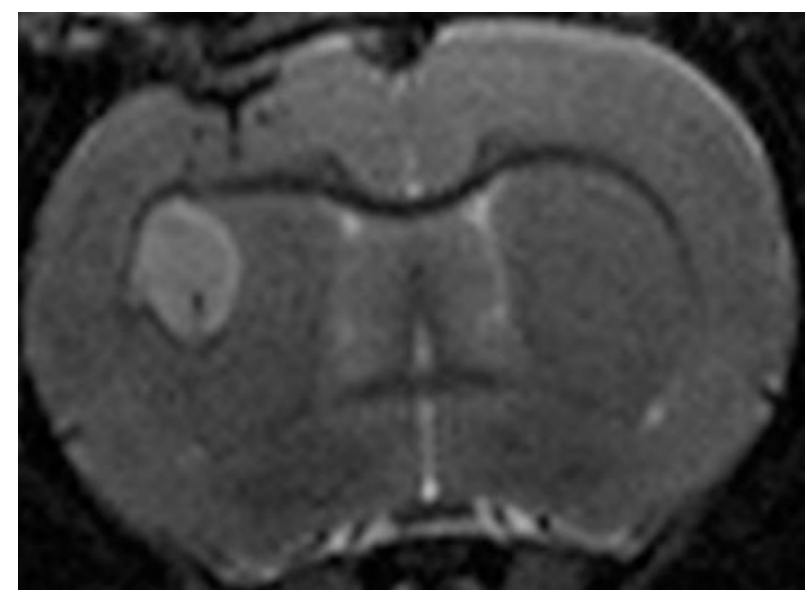

(a)

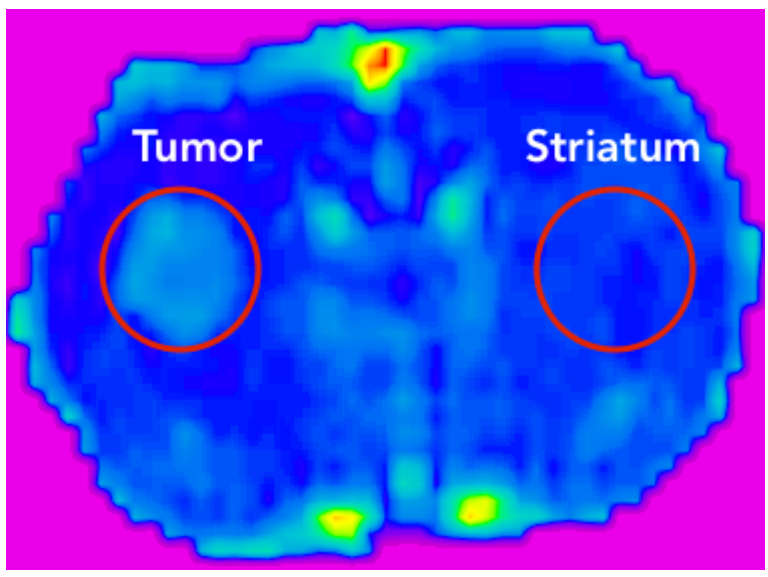

(c)
Post-treatment MRI

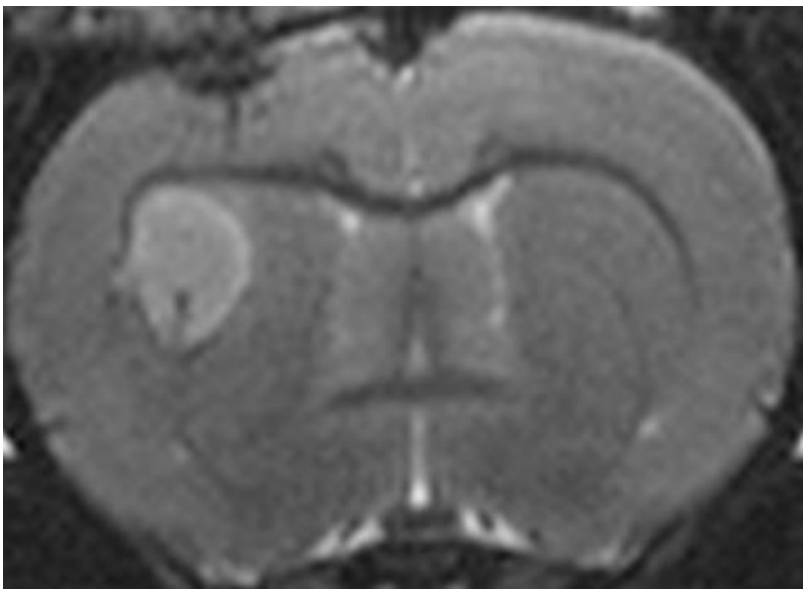

(b)

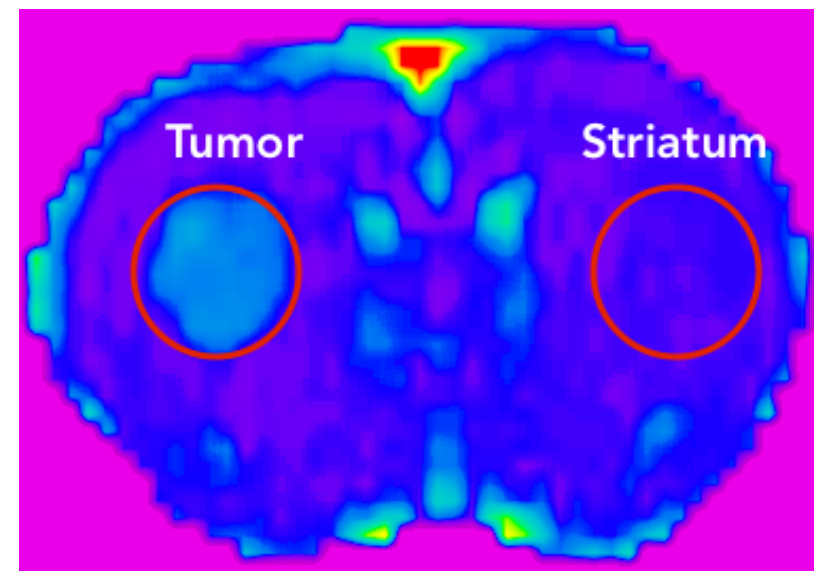

(d) 


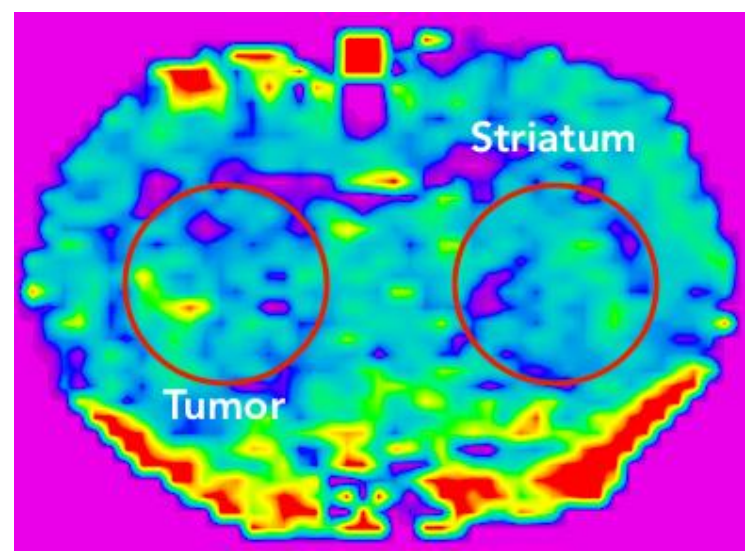

(e)

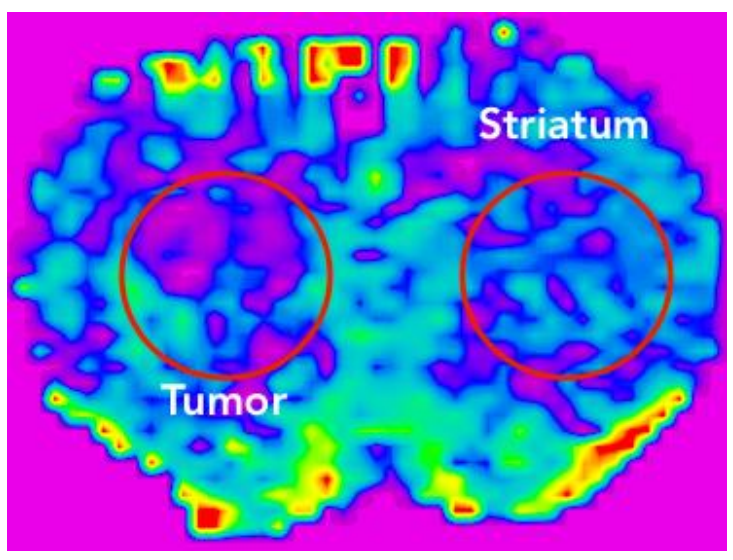

(f)

Figure 2: Pre- and post-operative MRI examinations of rat \#29, which belonged to the sham drug-only group. (a) Pre-operative and (b) post-operative T2 MRI; (c) Pre-operative and (d) Post-operative diffusion MRI; (e) Preoperative and (f) Post-operative perfusion MRI. Post-operative MRI was performed 72 hours after the introduction of the fiber. (a) and (b): T2, tumor slightly hyperintense without edema. (c) and (d): Slight increase in diffusion within the tumor. 
Pre-treatment MRI

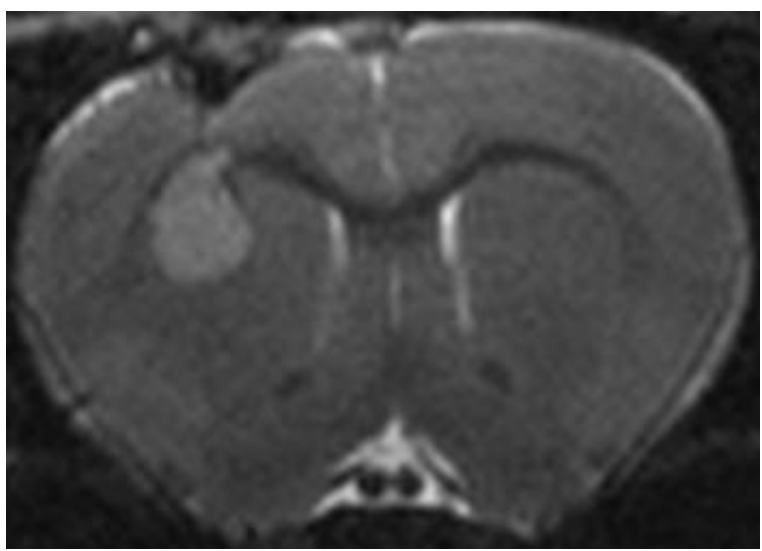

(a)

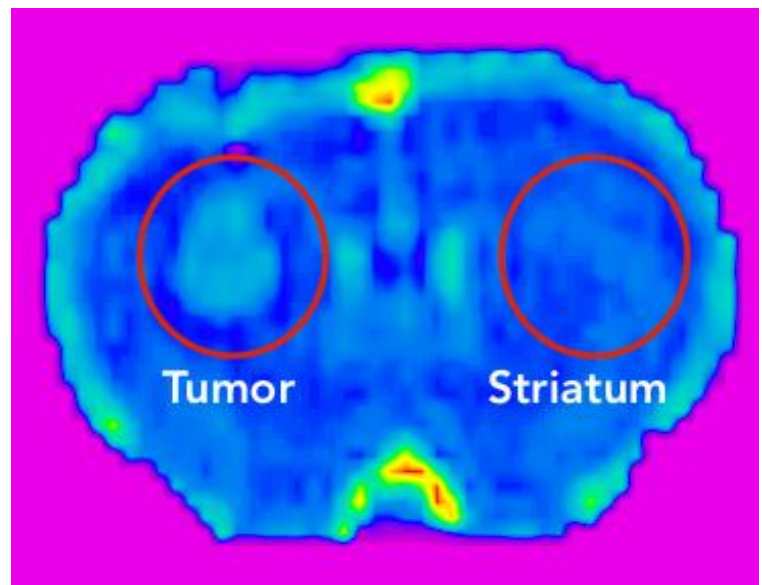

(c)

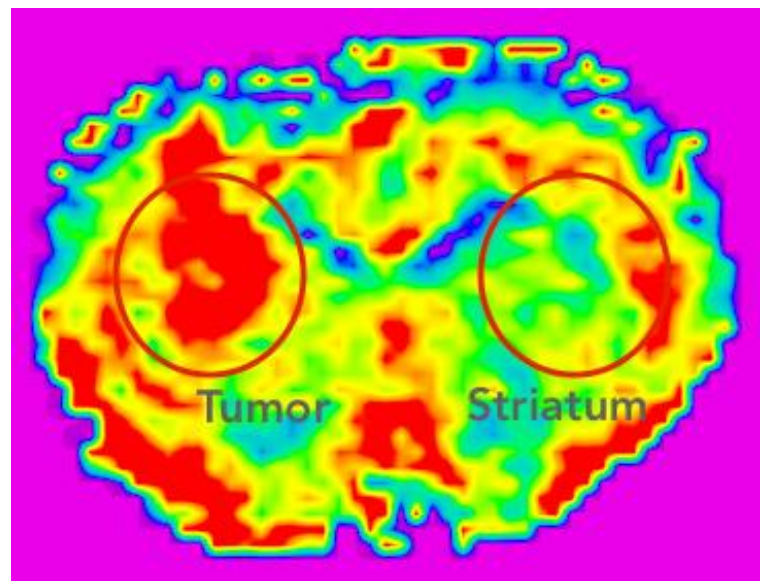

(e)
Post-treatment MRI

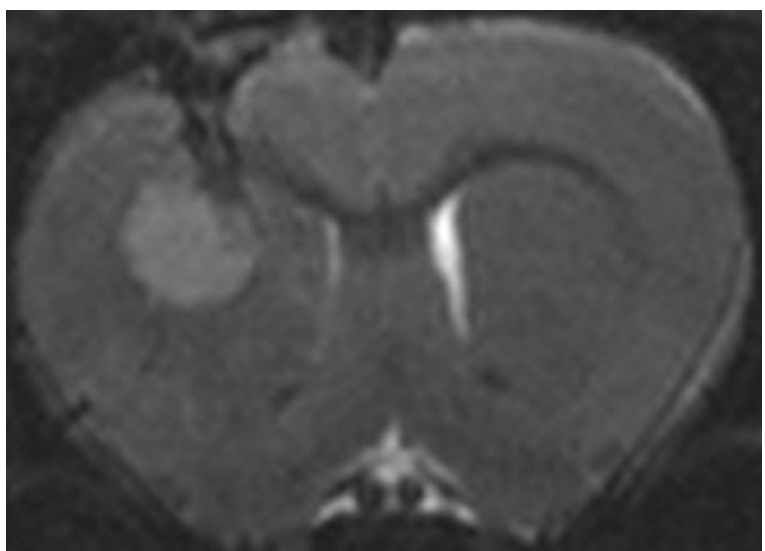

(b)

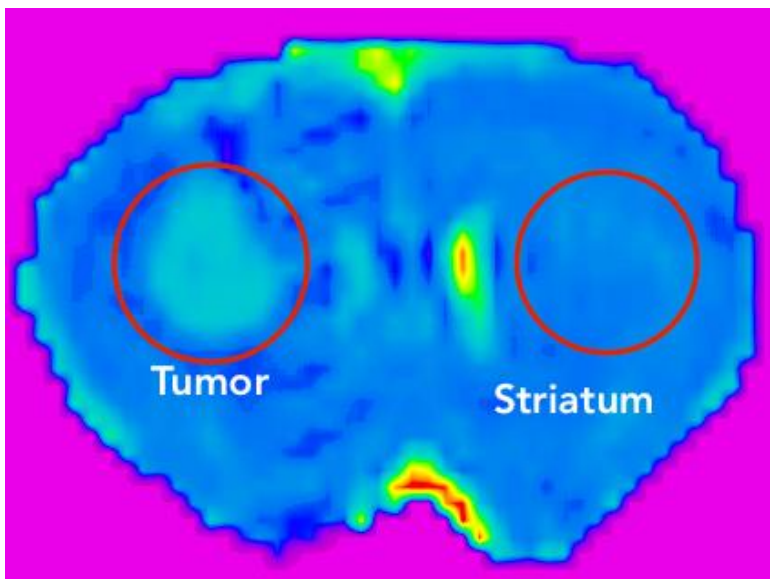

(d)

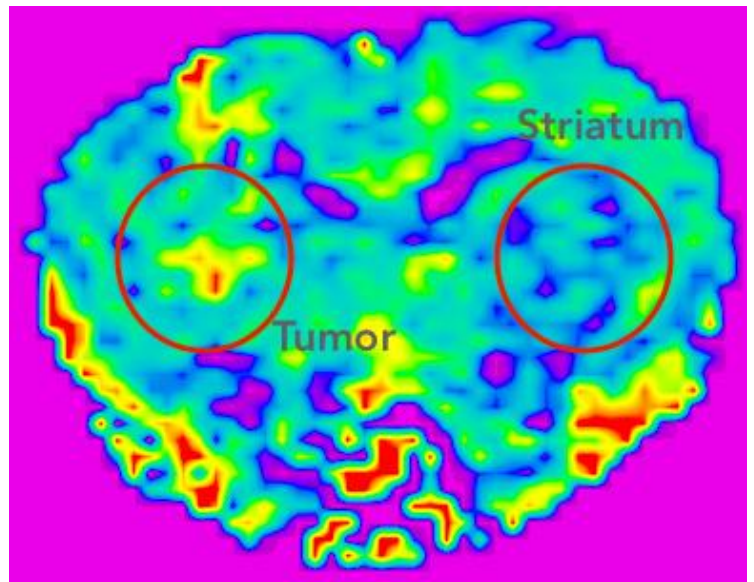

(f)

Figure 3: Pre- and post-operative MRI examinations of rat \#24, which belonged to the sham light-only group. (a) Pre-operative and (b) post-operative T2 MRI; (c) Pre-operative and (d) post-operative diffusion MRI; (e) Pre-operative and (f) post-operative perfusion MRI. Post-operative MRI was performed 72 hours after the introduction of the fiber. (a) and (b): T2, tumor slightly hyperintense without edema. (c) and (d): Slight increase in diffusion within the tumor. (e) and (f): Hyperperfusion of the tumor tissue and cortex. 
Pre-treatment MRI

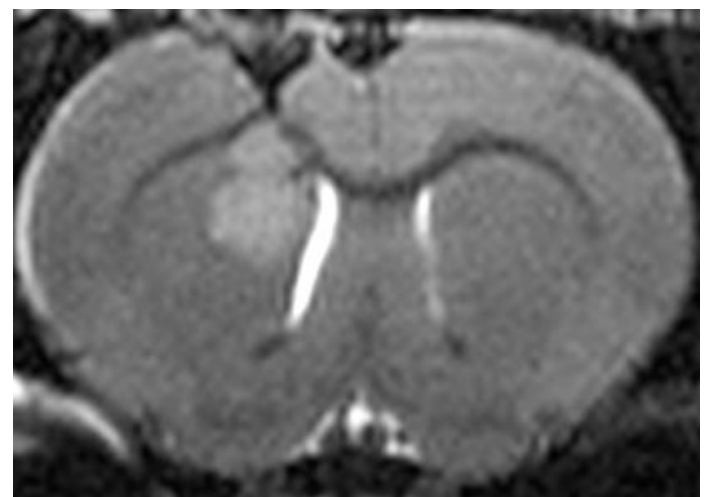

(a)

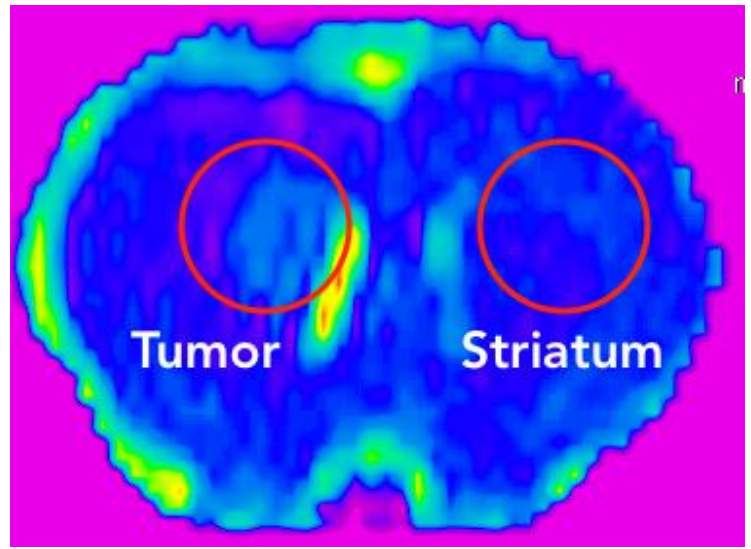

(c)

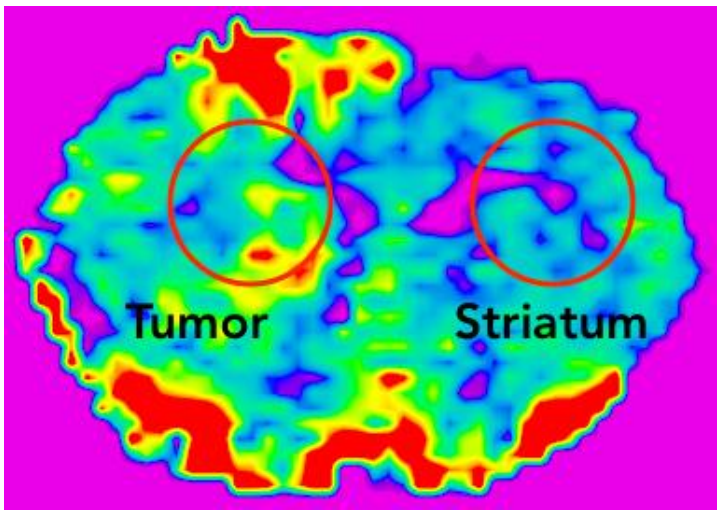

(e)
Post-treatment MRI

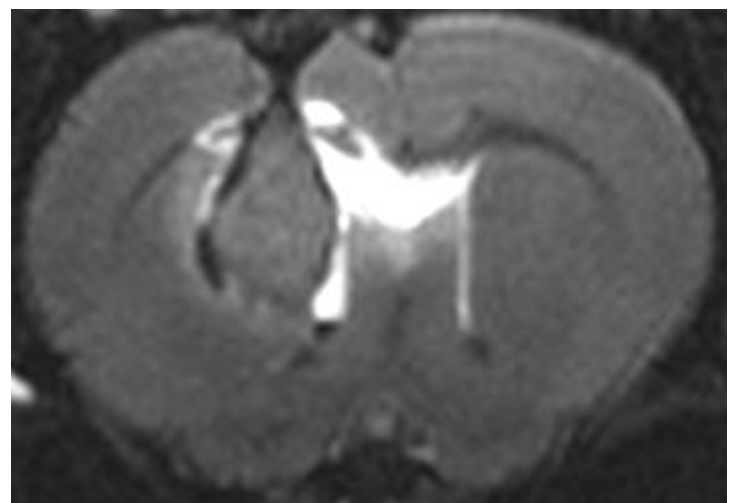

(b)

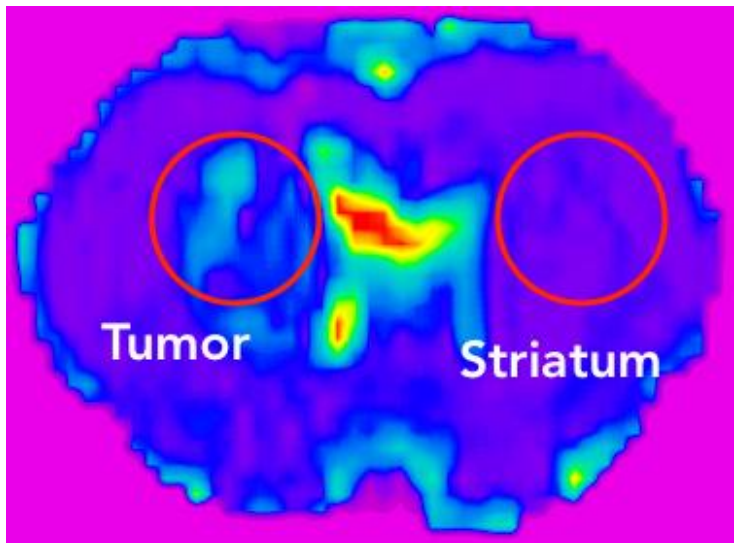

(d)

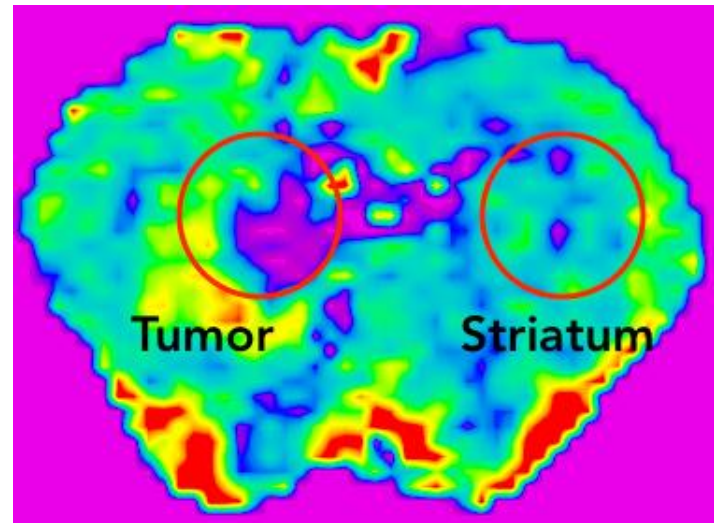

(f)

Figure 4: Pre- and post-operative MRI examinations of rat \#17, which belonged to the 2-fraction high-power group. (a) Pre-operative and (b) post-operative T2 MRI; (c) Pre-operative and (d) post-operative diffusion MRI; (e) Pre-operative and (f) Post-operative perfusion MRI. Post-operative MRI was performed 72 hours after the introduction after the 2 -fraction treatment at $30 \mathrm{~mW}$. (b versus a): T2 heterogeneous lesion against the right putamen, but not in the central part of the tumor; peritumoral edema visible after treatment (b). (d versus c): Increase in the diffusion coefficient within the tumor corresponding to necrosis induced by PDT. (f versus e): Increase in perfusion in the peritumoral edema contrasting with a sharp decrease in the central part of the tumor, confirming a reduction in tumoral angiogenesis induced by PDT (vascular necrosis). 
Pre-treatment MRI

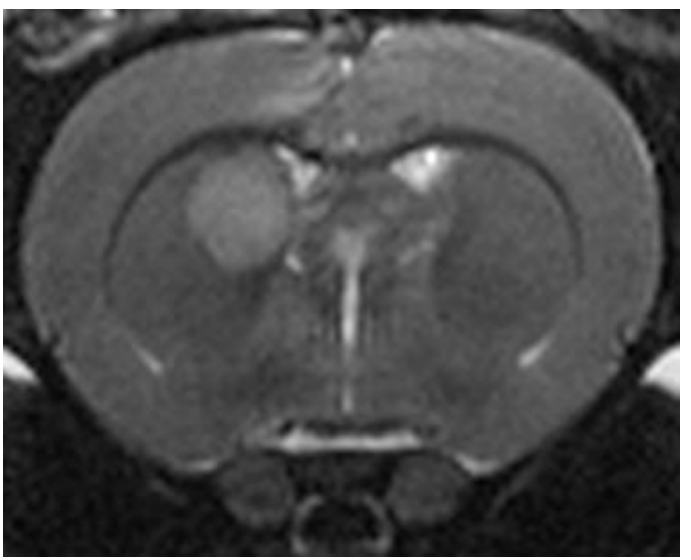

(a)

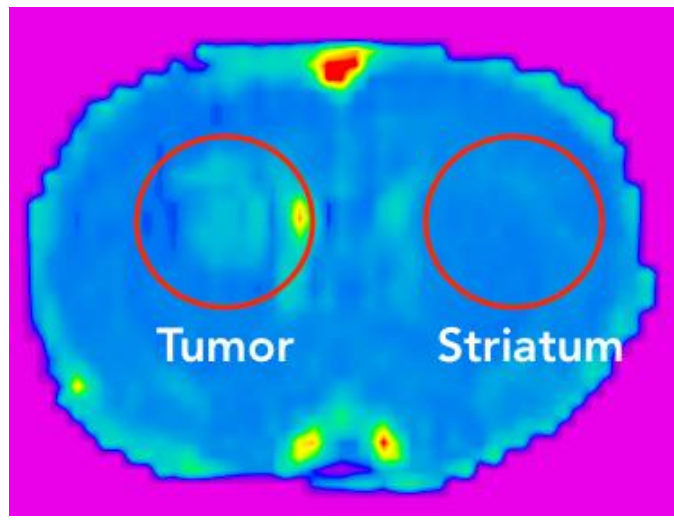

(c)

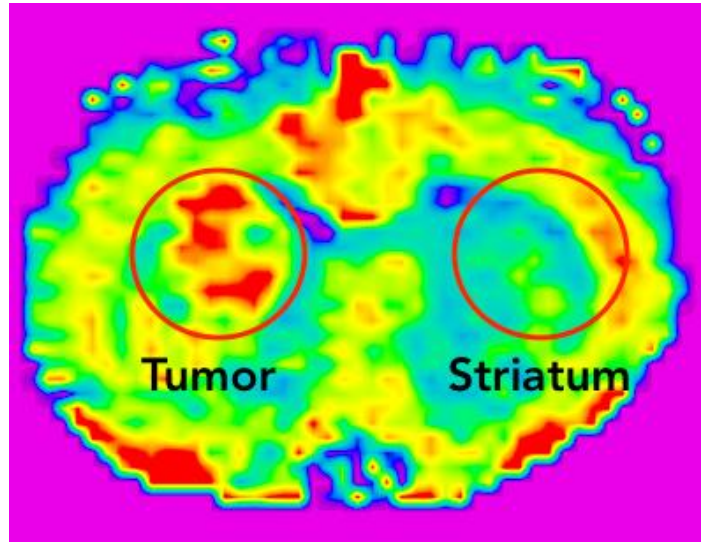

(e)
Post-treatment MRI

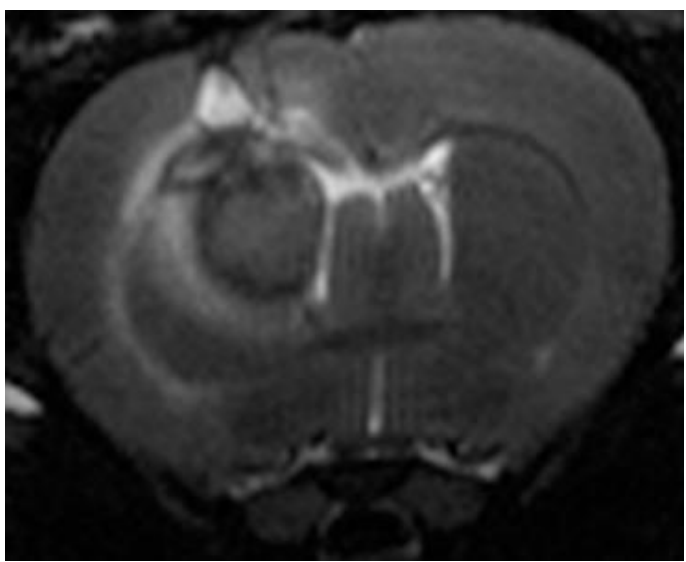

(b)

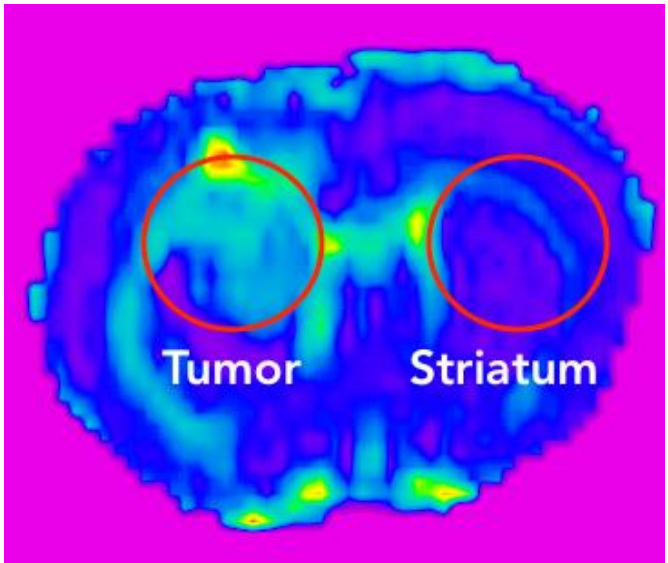

(d)

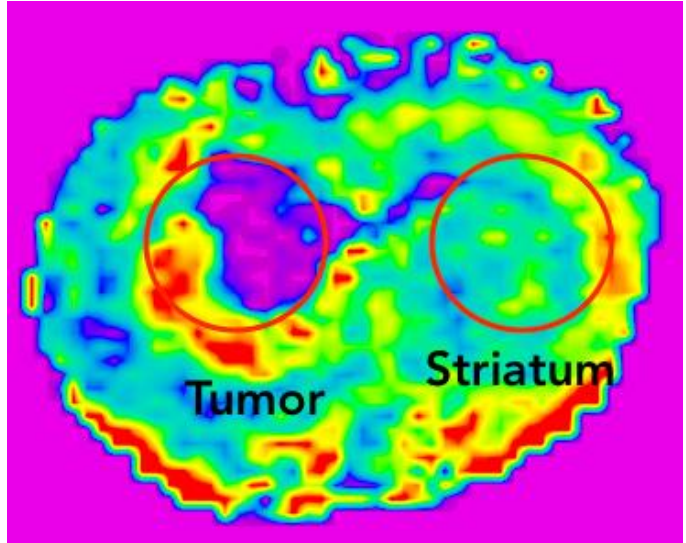

(f)

Figure 5: Pre- and post-operative MRI examinations of rat \#32, which belonged to the 2-fraction low-power group. (a) Pre-operative and (b) post-operative T2 MRI; (c) Pre-operative and (d) Post-operative diffusion MRI; (e) Pre-operative and (f) Post-operative perfusion MRI. Post-operative MRI was performed 72 hours after the 2-fraction treatment at $5 \mathrm{~mW}$. (b versus a): Large hyposignal area with peritumoral edema. (d versus c): Increase in the diffusion coefficient within the tumor corresponding to necrosis induced by PDT. (f versus e): Increase in perfusion in the peritumoral edema contrasting with a sharp decrease in the central part of the tumor, confirming vascular necrosis induced by PDT. 
Pre-treatment MRI

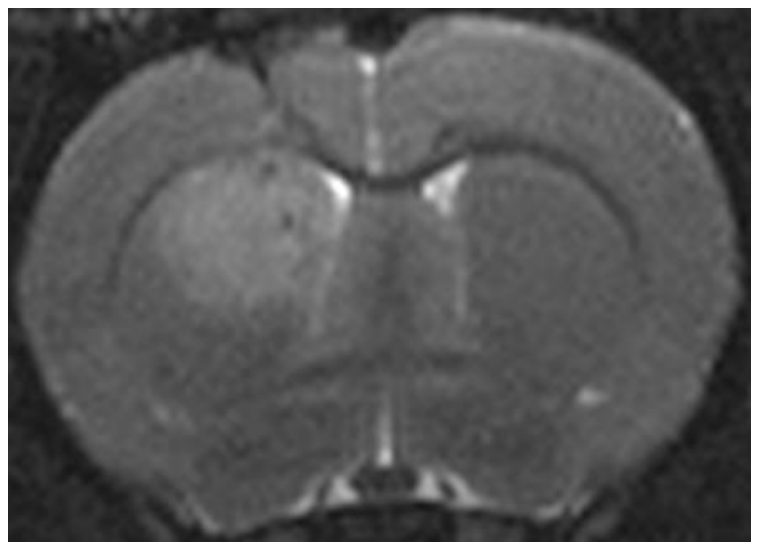

(a)

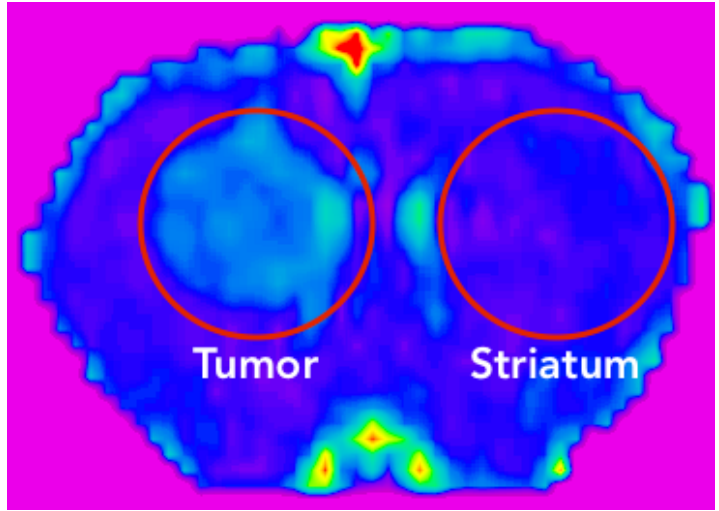

(c)

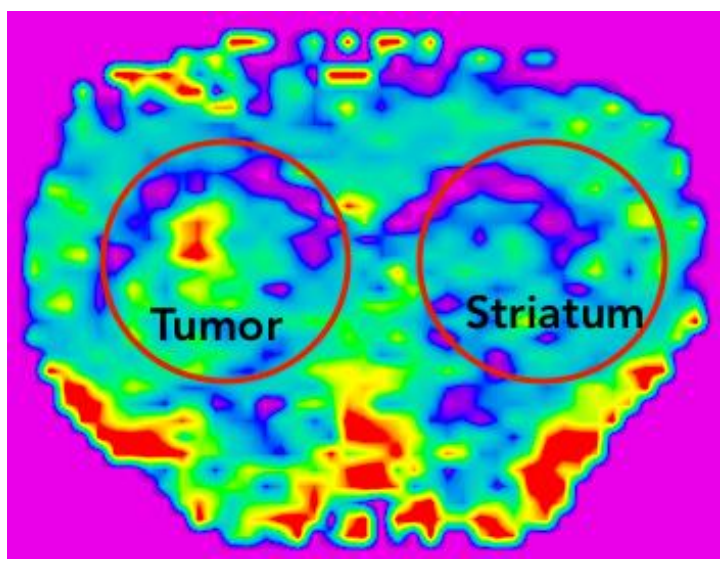

(e)
Post-treatment MRI

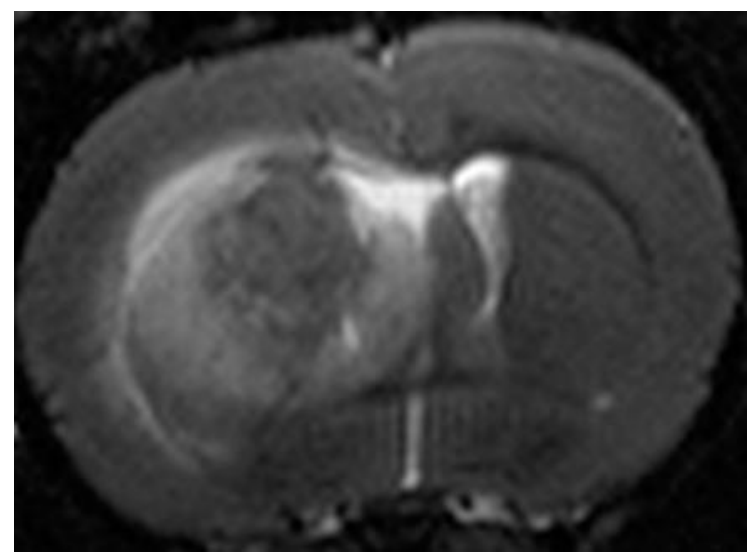

(b)

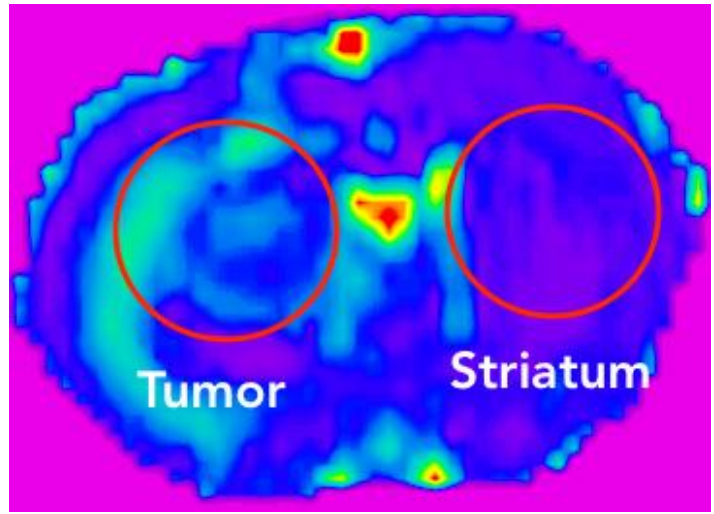

(d)

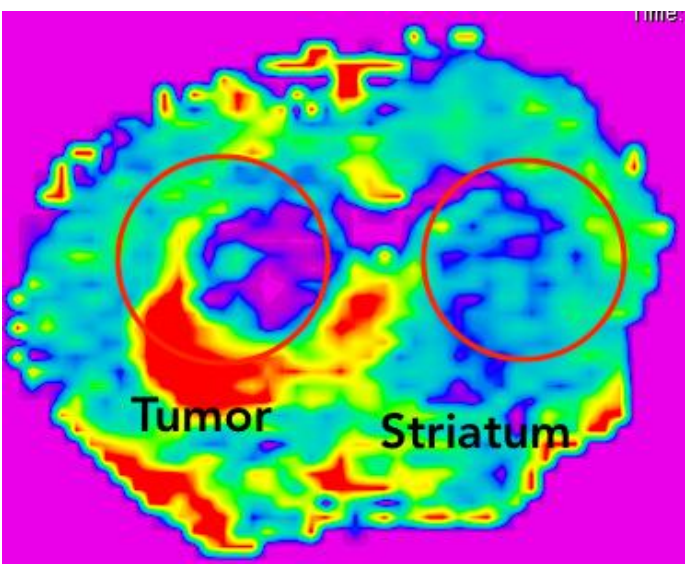

(f)

Figure 6: Pre- and post-operative MRI examinations of rat \#18, which belonged to the 5-fraction group treated at $30 \mathrm{~mW}$. (a) Pre-operative and (b) post-operative T2 MRI; (c) Pre-operative and (d) Post-operative diffusion MRI; (e) Pre-operative and (f) Post-operative perfusion MRI. Post-operative MRI was performed 72 hours after the 5-fraction treatment. (b versus a): Large hyposignal area with peritumoral edema. (d versus c): Slight increase in the diffusion coefficient in the center of the tumor corresponding to necrosis induced by PDT. (f versus e): Increased perfusion in the peritumoral edema contrasting with a sharp decrease in perfusion of the central part of the tumor, confirming vascular necrosis induced by PDT. 
Pre-treatment MRI

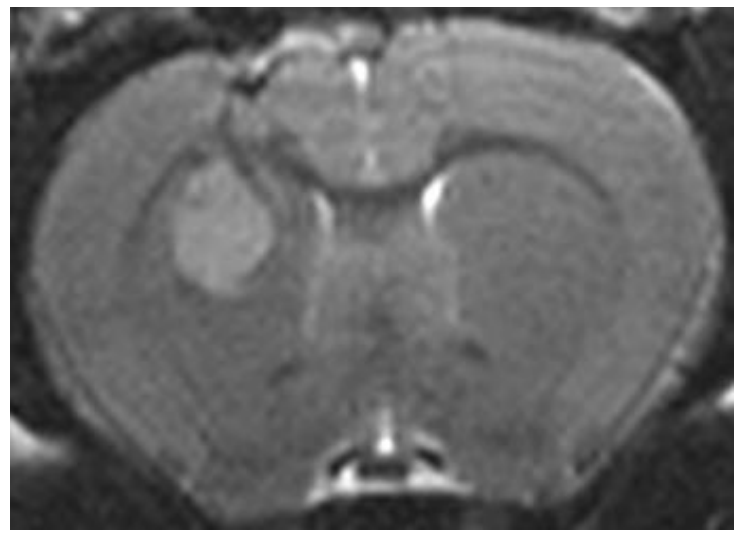

(a)

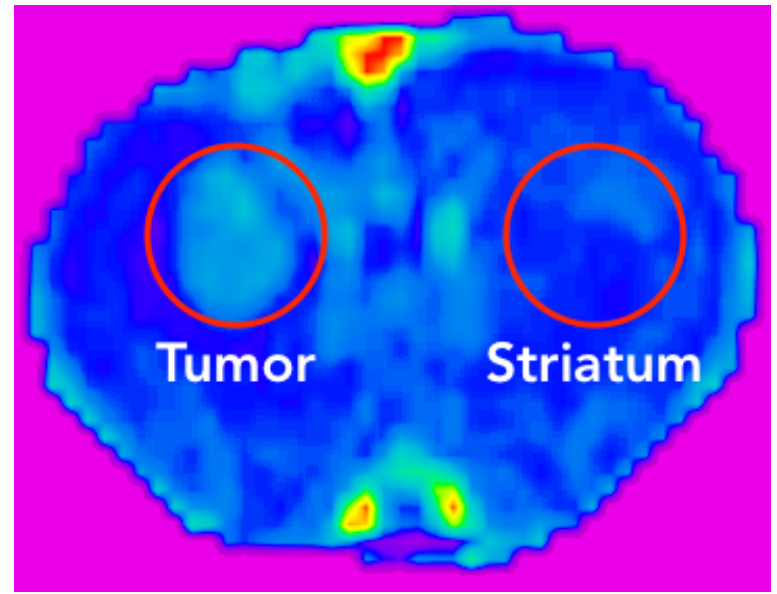

(c)

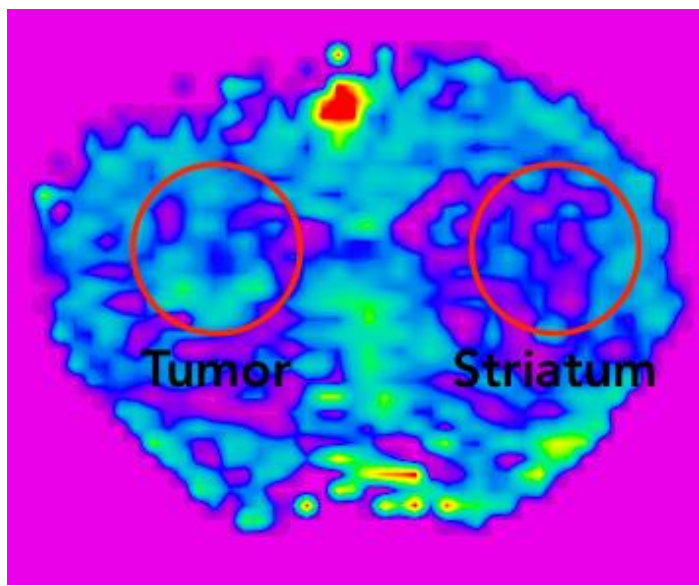

(e)
Post-treatment MRI

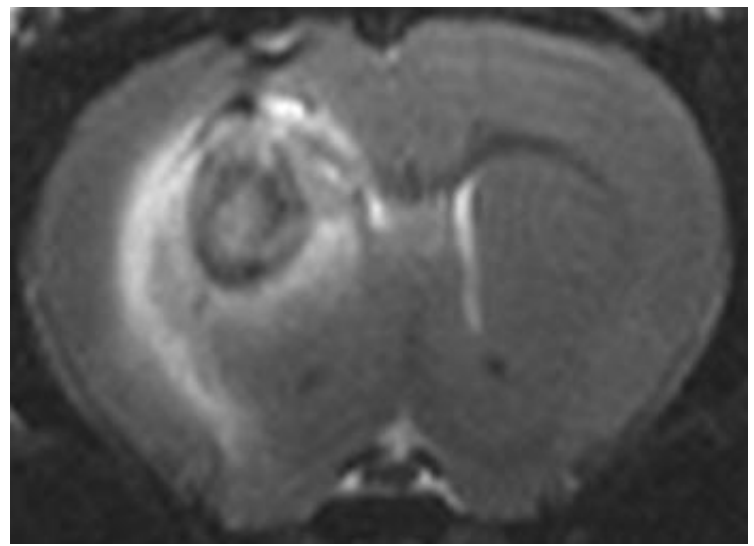

(b)

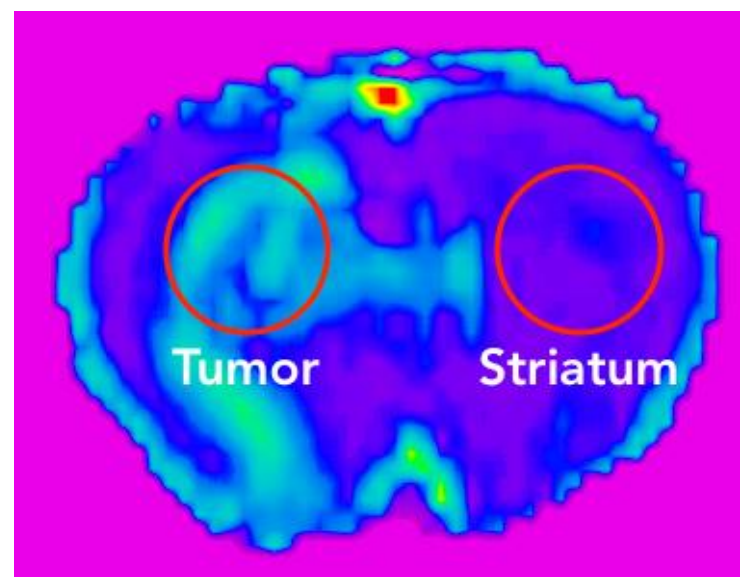

(d)

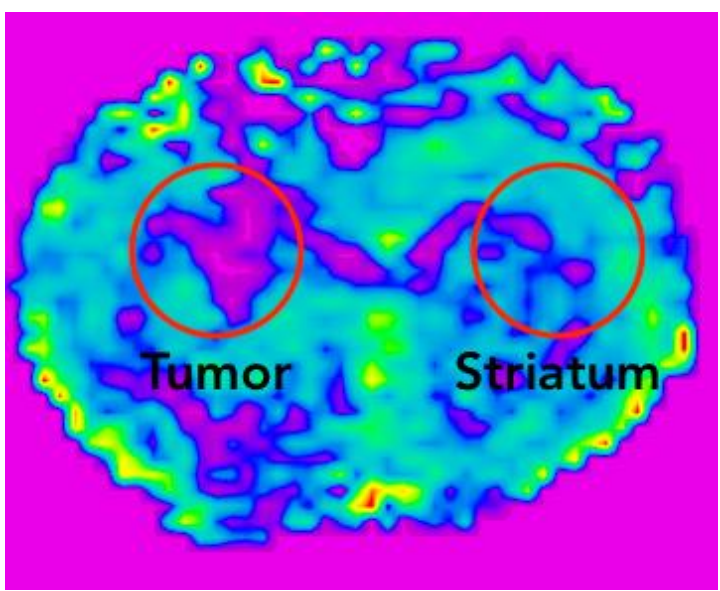

(f)

Figure 7: Pre- and post-operative MRI examinations of rat \#33, which belonged to the 5-fraction low-power group. (a) Pre-operative and (b) post-operative T2 MRI; (c) Pre-operative and (d) post-operative diffusion MRI; (e) Pre-operative and (f) Post-operative perfusion MRI. Post-operative MRI was performed 72 hours after the introduction of the fiber. (b versus a): Peritumoral edema visible on the T2 image (d versus c): Slight increase in the diffusion coefficient within the tumor corresponding to necrosis induced by PDT. (f versus e): Decrease in perfusion in the center of the tumor, confirming vascular necrosis induced by PDT. 
a) Diffusion

Forty-eight rats benefited from pre- and post-treatment diffusion sequences. All $\Delta \mathrm{d}$ values are represented in Figure 8 and summarized in Table 1.

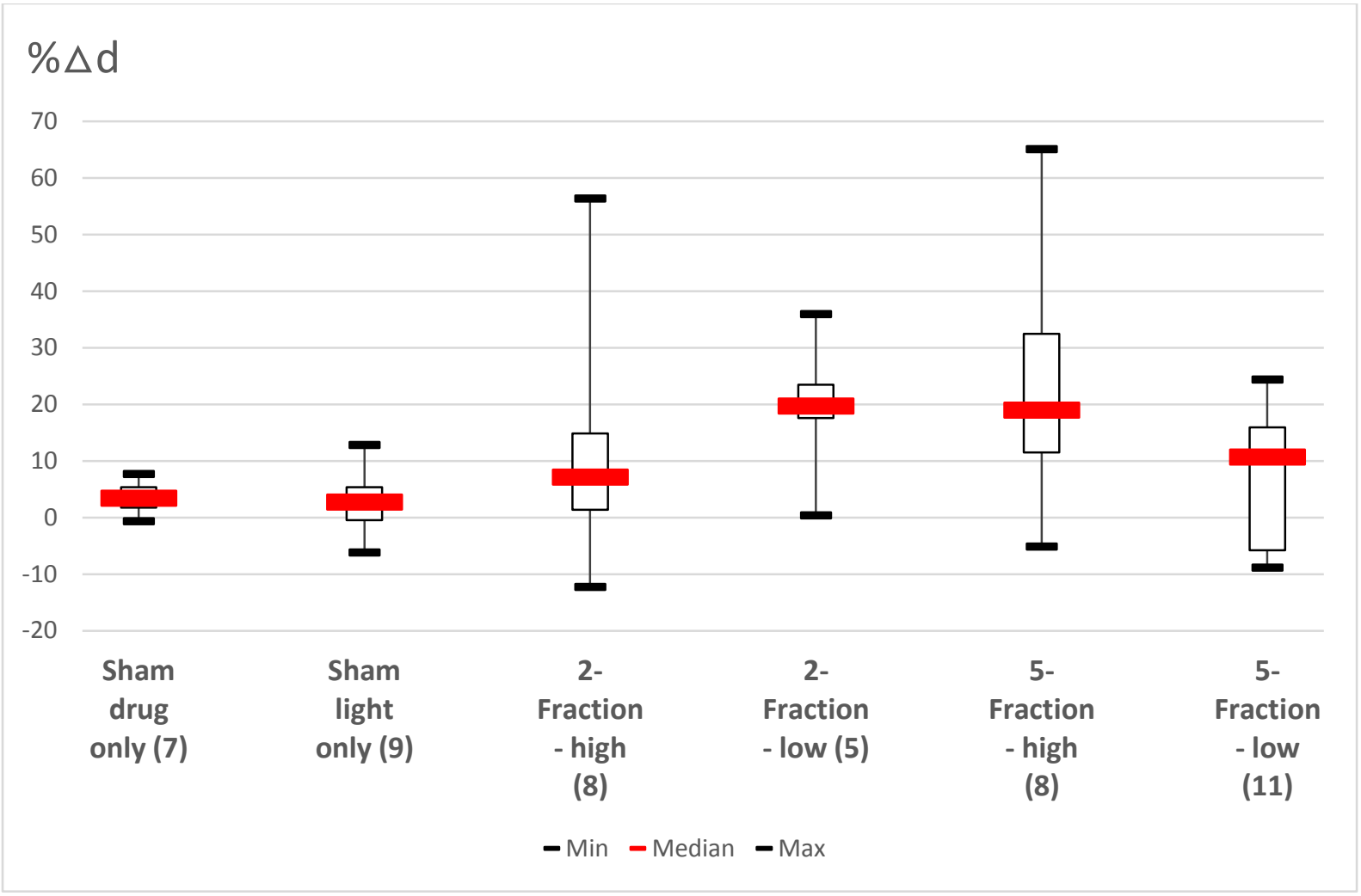

Figure 8: Boxplot illustrating $\Delta \mathrm{d}$ for the sham, 2-fraction and 5-fraction groups (red marks represent the median, the size of the box indicates the interquartile range (IQR), and the minimum/maximum are indicated by black marks). The number of specimens per group is shown in brackets.

There was no difference in $\Delta \mathrm{d}$ among the six groups ( $\mathrm{p}=0.0549$, Kruskal-Wallis test); however, the p-value was slightly higher than 0.05 , indicating a tendency toward a difference. In particular, $\Delta \mathrm{d}$ for the 2-fraction low-power, 5-fraction high-power and 5-fraction lowpower tended to be greater.

b) Perfusion

Among the forty-eight rats, forty-seven benefited from pre- and post-treatment perfusion sequences. All $\Delta \mathrm{p}$ values are represented in Figure 9 and summarized in Table 1. 


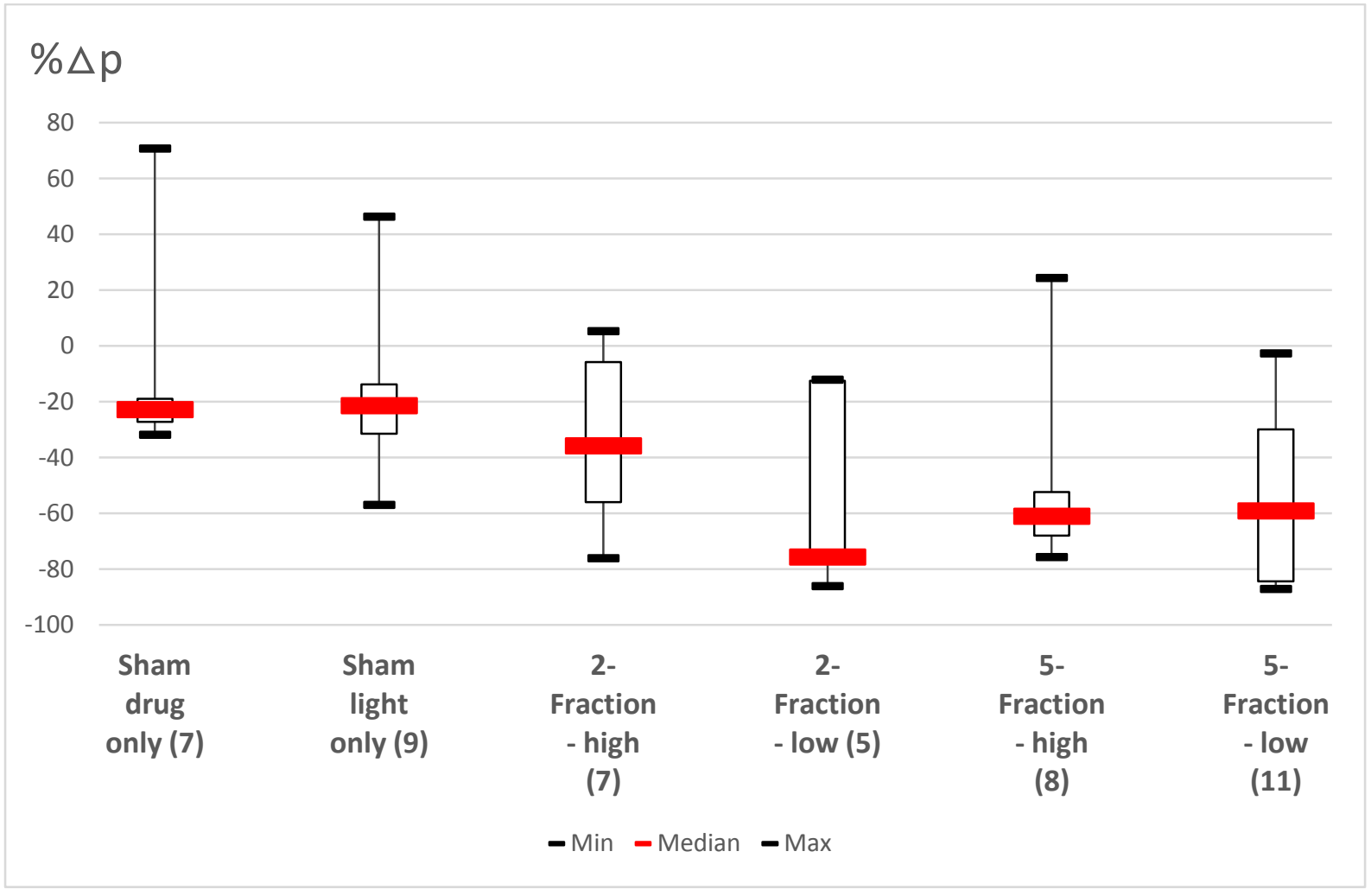

Figure 9: Boxplot illustrating $\Delta \mathrm{p}$ for the sham, 2-fraction and 5-fraction groups (red marks represent the median, the size of the box indicates the IQR, and the minimum/maximum are indicated by black marks). The number of specimens per group is shown in brackets.

There was a significant difference among the six groups in terms of the $\Delta p(p=0.048$, Kruskal-Wallis test), but no pairwise differences were found (due to the small sample sizes, pairwise comparison tests were not statistically powerful and therefore less likely to detect significant differences). However, when data in the 3 groups were pooled (sham, 2-fraction and 5-fraction), a significant difference was found ( $\mathrm{p}=0.0063$, Kruskal-Wallis test). The $\Delta \mathrm{p}$ was significantly lower for the 5-fraction group than for the sham group $(\mathrm{p}=0.002)$.

\section{c) Necrosis}

Among the forty-eight rats, forty-seven benefited from pre- and post-treatment T1 MRI sequences. All necrosis volumes are represented in Figure 10. 


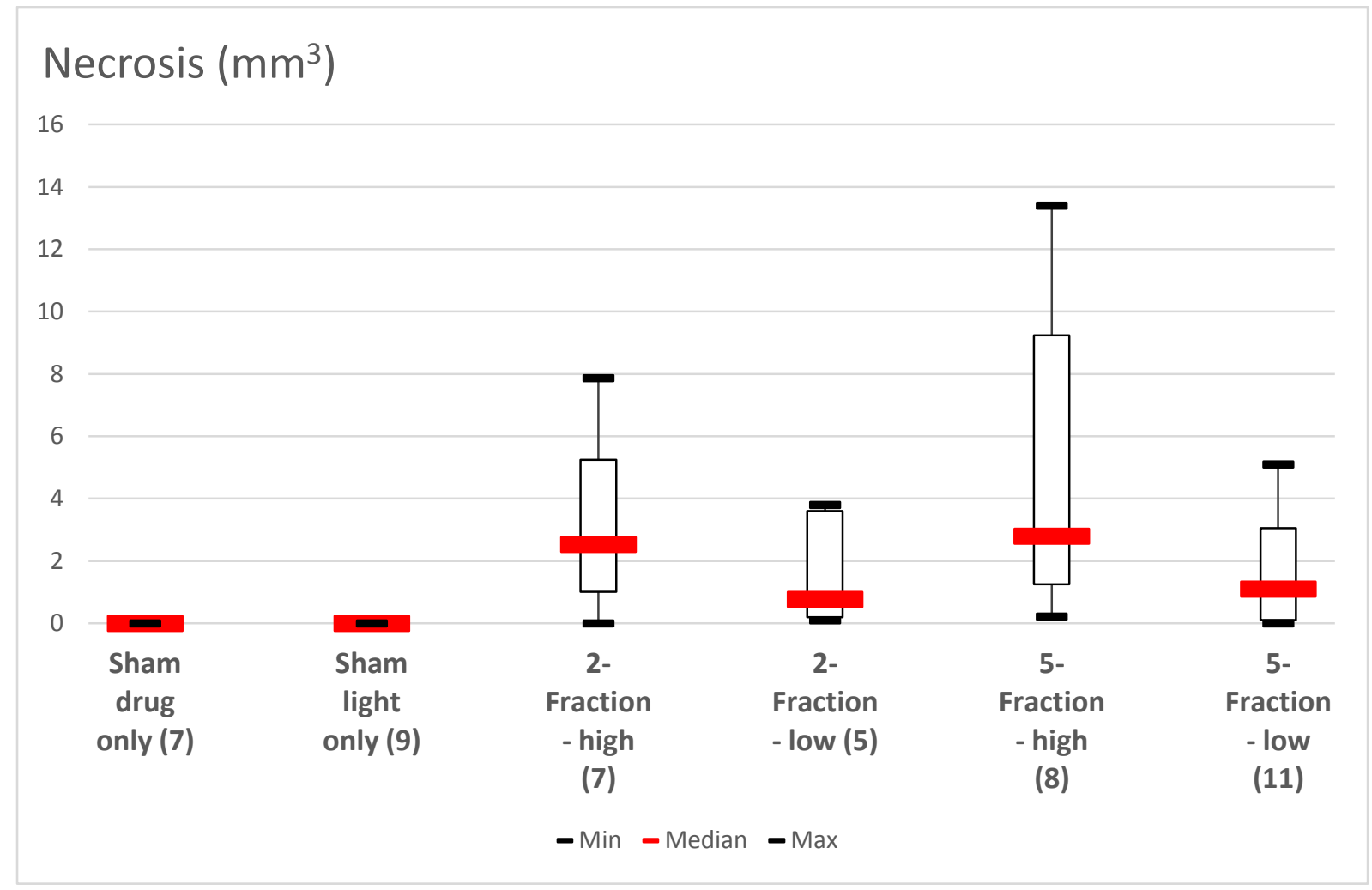

Figure 10: Boxplot illustrating the volume of necrosis in $\mathrm{mm}^{3}$ in the sham, 2-fraction and 5-fraction groups (red marks represent the median, the size of the box indicates the IQR, and the minimum/maximum are indicated by black marks). The number of specimens per group is shown in brackets.

The Kruskal-Wallis test followed by the Dwass-Steel-Critchlow-Fligner post hoc test revealed that the two sham groups had significantly smaller necrosis volumes than were observed in the four treatment groups ( $\mathrm{p}$-values ranging from 0.002 to 0.044 ). There were no significant differences between the two sham groups or among the four treatment groups (all p-values > $0.40)$.

d) Peritumoral edema volume

Among the forty-eight rats, forty-seven benefited from pre- and post-treatment T2 MRI sequences. All edema volumes are represented in Figure 11. 


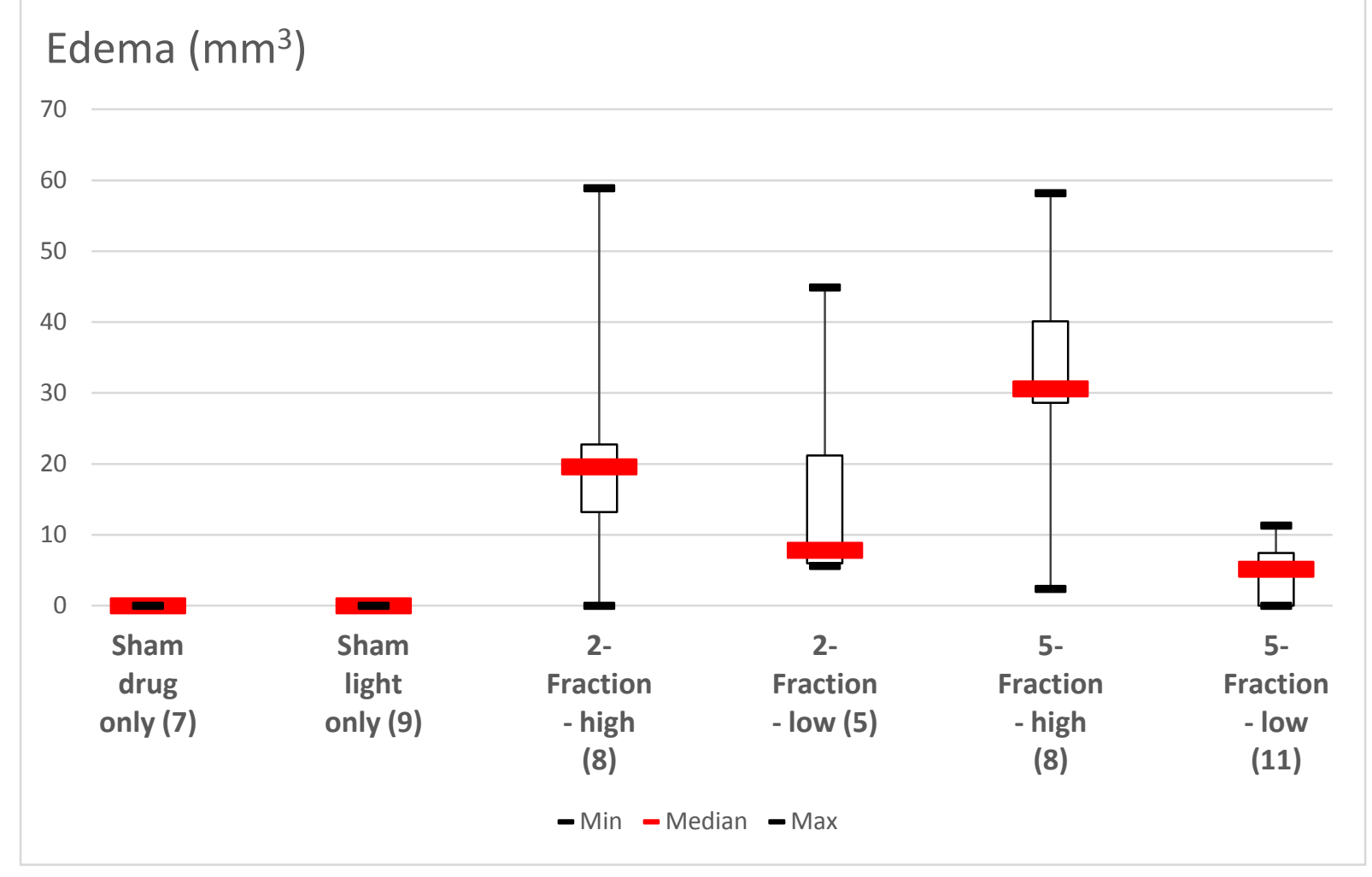

Figure 11: Boxplot illustrating edema volume in $\mathrm{mm}^{3}$ in the sham, 2-fraction and 5-fraction groups (red marks represent the median, the size of the box indicates the IQR, and the minimum/maximum are indicated by black marks). The number of specimens per group is shown in brackets.

Using the Kruskal-Wallis test followed by the Dwass-Steel-Critchlow-Fligner post hoc test, we found that the sham groups had significantly smaller edema volumes than were observed in the treatment groups (the two 2-fraction groups treated at $5 \mathrm{~mW}$ or $30 \mathrm{~mW}$ and the 5fraction group treated at $30 \mathrm{~mW}$ ) (p-values ranging from 0.002 to 0.05 ). Moreover, the 5fraction group treated with $30 \mathrm{~mW}$ was associated with edema volumes significantly larger than those observed in the 5-fraction group treated with $5 \mathrm{~mW}(\mathrm{p}=0.019)$. No other significant pairwise differences were found (all p-values higher than 0.05). 
Table 1 summarizes the specimens included per group, with the measures of each parameter reported for each group.

\begin{tabular}{|c|c|c|c|c|c|c|}
\hline & \multicolumn{2}{|c|}{ Sham } & \multicolumn{2}{|c|}{ 2-Fraction } & \multicolumn{2}{|c|}{ 5-Fraction } \\
\hline & Drug only & Light only & $\begin{array}{c}\text { @ } 30 \text { mW } \\
\text { (high) }\end{array}$ & $\begin{array}{c}\text { @ } 5 \text { mW } \\
\text { (low) }\end{array}$ & $\begin{array}{c}\text { @ } 30 \text { mW } \\
\text { (high) }\end{array}$ & $\begin{array}{c}\text { @5 mW } \\
\text { (low) }\end{array}$ \\
\hline $\begin{array}{l}\text { Specimen } \\
\text { number }\end{array}$ & 8 & 9 & 8 & 5 & 8 & 11 \\
\hline $\begin{array}{l}\Delta d \% \\
(I Q R)\end{array}$ & $\begin{array}{c}3.41 \% \\
(1.75,5.39)\end{array}$ & $\begin{array}{c}2.73 \% \\
(-0.45,5.37)\end{array}$ & $\begin{array}{c}7.14 \% \\
(1.36,14.88)\end{array}$ & $\begin{array}{c}19.70 \% \\
(17.59,23.48)\end{array}$ & $\begin{array}{c}18.98 \% \\
(11.51,32.46)\end{array}$ & $\begin{array}{c}10.69 \% \\
(-5.75,15.95)\end{array}$ \\
\hline $\begin{array}{l}\Delta p \% \\
(I Q R)\end{array}$ & $\begin{array}{c}-22.77 \% \\
(-27.17,- \\
19.00)\end{array}$ & $\begin{array}{c}-21.36 \% \\
(-31.52,- \\
13.77)\end{array}$ & $\begin{array}{c}-35.75 \% \\
(-56.00,-5.81)\end{array}$ & $\begin{array}{c}-75.61 \% \\
(-76.19,- \\
12.53)\end{array}$ & $\begin{array}{c}-61.00 \% \\
(-67.99,- \\
52.35)\end{array}$ & $\begin{array}{c}-59.08 \% \\
(-84.35,29.8)\end{array}$ \\
\hline $\begin{array}{c}\text { Necrosis } \\
\left(\mathbf{m m}^{3}\right)\end{array}$ & $\begin{array}{c}0 \\
(0,0)\end{array}$ & $\begin{array}{c}0 \\
(0,0)\end{array}$ & $\begin{array}{c}2.53 \\
(1.00,5.24)\end{array}$ & $\begin{array}{c}0.77 \\
(0.2,3.6)\end{array}$ & $\begin{array}{c}2.79 \\
(1.25,9.24)\end{array}$ & $\begin{array}{c}1.1 \\
(0.1,3.05)\end{array}$ \\
\hline $\begin{array}{c}\text { Edema } \\
\left(\mathbf{m m}^{3}\right)\end{array}$ & $\begin{array}{c}0 \\
(0,0)\end{array}$ & $\begin{array}{c}0 \\
(0,0)\end{array}$ & $\begin{array}{c}19.6 \\
(13.2,22.72)\end{array}$ & $\begin{array}{c}21.17 \\
(7.82,44.90)\end{array}$ & $\begin{array}{c}30.6 \\
(28.62,40.1)\end{array}$ & $\begin{array}{c}5.15 \\
(0,7.43)\end{array}$ \\
\hline
\end{tabular}

Table 1: Number of specimens eligible for PDT that underwent post-treatment MRI per group (row 2) and summary of $\Delta \mathrm{d}$ and $\Delta \mathrm{p}$ values and necrosis and edema volumes.

\section{Discussion}

PDT is an emerging cancer treatment that is actively being studied in different body locations. Optimizing PDT delivery can be undertaken by either increasing 5-ALA uptake and PpIX accumulation(23) or considering different light regimens that affect tissue oxygenation and may influence free radical production and oxidative species formation $(7,8)$.

In a previous study(12), we found that diffusion and perfusion sequences accurately assessed iPDT effects, with diffusion being a relevant marker of cell death and perfusion indicating the level of necrosis. The objective of the current study was to compare previous findings obtained when iPDT was delivered at $30 \mathrm{~mW}$ with a new light protocol performed with delivery at a lower power $(5 \mathrm{~mW})$. Thus, tumor cell death was evaluated by diffusion 
imaging, necrosis levels were evaluated by T1 MRI, and perfusion imaging was evaluated for six different treatment schemes. Edema was measured on T2 imaging to grade the toxicity of the treatment delivery strategy.

With regard for diffusion imaging acquired before and after iPDT, $\Delta \mathrm{d}$ was not significantly different among the six groups, but there was a tendency toward a difference (p-value slightly greater than 0.05 ( $\mathrm{p}=0.0549$, Kruskal-Wallis test)). In particular, the 2-fraction low, 5-fraction high and 5-fraction low schemes tended to lead to greater $\Delta \mathrm{d}$. This observation is in line with a previously proposed assumption that the consumption of oxygen influences the photochemical reaction $(10,12,19)$. Indeed, too much oxygen was probably consumed when light was delivered in only two fractions at a high power. The photochemical reaction was thus limited regardless of the total energy deposited. The importance of fractionation has already been emphasized by Curnow et al. (8)., who measured the level of oxygen through an oxygen microelectrode to study the effect of PDT delivered with continuous or fractionated light regimens. They found that the level of tissue oxygen at the treatment site was differentially affected according to light regimens and that this affected the treatment effects. Those findings are corroborated by our own observations obtained using diffusion imaging, which showed that the best configuration tended to be 5 fractions delivered at $5 \mathrm{~mW}$.

Concerning perfusion imaging, there was a significant difference among the six groups in terms of $\Delta \mathrm{p}(\mathrm{p}=0.048$, Kruskal-Wallis test), but there were no pairwise differences. However, a significant pairwise difference was found $(\mathrm{p}=0.0063$, Kruskal-Wallis test) when the three pooled groups were considered: sham, 2-fraction and 5-fraction (regardless of the light power). $\Delta \mathrm{p}$ for 5 fractions was significantly lower than that observed in the sham group $(\mathrm{p}=0.002)$, indicating that 5 fractions led to a more significant effect. These results support the notion that PDT exerts a lesional effect on the tumor vasculature, corroborating the decrease in perfusion values observed within the treated tumors. Additionally, Leroy et al. (10) found that vascular structures were no longer observed within the necrotic areas on histological analysis, corroborating that there was a decrease in perfusion values within the treated tumors when necrosis was measured directly on T1 MRI (Figure 10). In fact, significant pairwise differences were also found in terms of necrosis between the treated and sham groups, but there was no significant difference between the 5 -fraction $5 \mathrm{~mW}$ group and the 5-fraction 30 $\mathrm{mW}$ group. Similarly, in Curnow et al.(8), the authors found that fractionation led to up to three times more necrosis than no fractionation when a constant energy deposit was assumed. 
The toxicity of the treatment modality (i.e., fractions and light power) was estimated by measuring the volume of edema on T2 MRI. The sham groups had significantly smaller edema volumes than those observed in the two 2-fraction groups and the 5-fraction group treated at $30 \mathrm{~mW}$ (p-values from 0.002 to 0.05 ) but were not significantly different from those observed in the 5 -fraction $5 \mathrm{~mW}$ group. Additionally, the 5 -fraction $5 \mathrm{~mW}$ group was associated with significantly lower edema volumes than were found in the 5-fraction $30 \mathrm{~mW}$ group ( $\mathrm{p}=0.019$ ), indicating that this treatment modality had a lower toxicity and thus was better tolerated.

According to MRI data and analyses aimed at balancing the treatment effect (level of necrosis by cell deaths characterized by diffusion imaging) and treatment toxicity, the 5 -fraction regimen delivered at $5 \mathrm{~mW}$ was the most efficient. Indeed, if the 2-fraction treatment at 30 $\mathrm{mW}$ is a good candidate for minimizing necrosis, toxicity scored by the edema level was higher than that in the 5 -fraction group at $5 \mathrm{~mW}$. Additionally, $\Delta \mathrm{p}$ was lower in the 5 -fraction group at $5 \mathrm{~mW}$ than that measured in the 5 -fraction group at $30 \mathrm{~mW}$, indicating a more pronounced lesional effect.

However, even if MRI allowed a rapid treatment effect analysis, a comprehensive study of the tumor cell death path according to the treatment modality is highly expected to further optimize PDT delivery. Indeed, this preliminary study was conducted in a small number of animals per group and could form a basis for a more complete set of experiments, including oxygen level measurements(8), staining for new vessels to explore vascular density (e.g., CD31) and apoptosis staging (e.g., with the TUNEL method, ApopTag® Plus Peroxidase In Situ, Millipore, USA).

Finally, transposing the notion of "high" and "low" energy to humans remains highly challenging. Indeed, the fractionation scheme is easily transposed to humans and has already been proposed in the clinical trial INDYGO (24). However, evaluating the effect of light power is complex, and the choice of optimal power should be carefully balanced. Indeed, the time needed for therapeutic energy deposit may drastically increase the surgery procedure duration. Data collected during the INDYGO trial might help to clarify this question.

\section{Conclusion}

We previously reported that MRI examinations, including diffusion and perfusion sequences, can accurately assess iPDT treatment effects. In the current study, we applied the same MRI 
imaging protocol to a larger population composed of specimens analyzed in our previous study (i.e., 2- and 5-fractions delivered at $30 \mathrm{~mW}$ ) and new specimens treated according to different modalities (i.e., 2- and 5-fractions delivered at $5 \mathrm{~mW}$ ). From observations obtained from MRI data and in consideration of treatment effects (level of necrosis as cell deaths characterized by diffusion imaging) and treatment toxicity, the 5-fraction treatment delivered at $5 \mathrm{~mW}$ was the most efficient treatment scheme. In this context, this group might be a good candidate for a more comprehensive exploration of cell death pathways following the delivery of PDT to tumor cells. 


\section{References:}

1. Iacob G, Dinca EB. Current data and strategy in glioblastoma multiforme. J Med Life. 2009;2(4):386-93.

2. Ostrom QT, Bauchet L, Davis FG, Deltour I, Fisher JL, Langer CE, et al. The epidemiology of glioma in adults: a "state of the science" review. Neuro Oncol. 2014;16(7):896-913.

3. Stupp R, Mason WP, van den Bent MJ, Weller M, Fisher B, Taphoorn MJ, et al. Radiotherapy plus concomitant and adjuvant temozolomide for glioblastoma. N Engl J Med. 2005;352(10):987-96.

4. Grill J. Glioblastoma 2007 [Available from: http://www.orpha.net/consor4.01/www/cgi-

bin/Disease_Search.php?Ing=EN\&data_id=3752\&Disease_Disease_Search_diseaseGroup=gl ioblastome\&Disease_Disease_Search_diseaseType=Pat\&Maladie(s)/groupes\%20de\%20mala $\underline{\text { dies }=\text { Glioblastome \&title }=\text { Glioblastome } \& \text { search }=\text { Disease_Search_Simple. }}$.

5. Fisher JL, Schwartzbaum JA, Wrensch M, Wiemels JL. Epidemiology of brain tumors. Neurol Clin. 2007;25(4):867-90, vii.

6. Stupp R, Brada M, van den Bent MJ, Tonn JC, Pentheroudakis G, Group EGW. Highgrade glioma: ESMO Clinical Practice Guidelines for diagnosis, treatment and follow-up. Ann Oncol. 2014;25 Suppl 3:iii93-101.

7. Henderson BW, Dougherty TJ. How does photodynamic therapy work? Photochem Photobiol. 1992;55(1):145-57.

8. Curnow A, Haller JC, Bown SG. Oxygen monitoring during 5-aminolaevulinic acid induced photodynamic therapy in normal rat colon. Comparison of continuous and fractionated light regimes. Journal of photochemistry and photobiology B, Biology. 2000;58(2-3):149-55.

9. Curnow A, McIlroy BW, Postle-Hacon MJ, MacRobert AJ, Bown SG. Light dose fractionation to enhance photodynamic therapy using 5-aminolevulinic acid in the normal rat colon. Photochemistry and photobiology. 1999;69(1):71-6.

10. Leroy HA, Vermandel M, Vignion-Dewalle AS, Leroux B, Maurage CA, Duhamel A, et al. Interstitial photodynamic therapy and glioblastoma: Light fractionation in a preclinical model. Lasers in surgery and medicine. 2017;49(5):506-15.

11. Tetard MC, Reyns N, Leroy HA, Lejeune JP, Mordon S, Vermandel M, editors. Light Dose Fractionation to Enhance Photodynamic Therapy With 5-Aminolevulinic Acid in Human glioma xenografts. The International Congress on Photodynamic Applications (ICPA 2014) 2014; Dundee, Scotland.

12. Leroy HA, Vermandel M, Leroux B, Duhamel A, Lejeune JP, Mordon S, et al. MRI assessment of treatment delivery for interstitial photodynamic therapy of high-grade glioma in a preclinical model. Lasers in surgery and medicine. 2018;50(5):460-8.

13. Angell-Petersen E, Hirschberg H, Madsen SJ. Determination of fluence rate and temperature distributions in the rat brain; implications for photodynamic therapy. Journal of biomedical optics. 2007;12(1):14003.

14. Henderson BW, Busch TM, Snyder JW. Fluence rate as a modulator of PDT mechanisms. Lasers in surgery and medicine. 2006;38(5):489-93.

15. Alqawi O, Espiritu M, Singh G, editors. Molecular mechanisms associated with ALAPDT of brain tumor cells2009.

16. Lilge L, Portnoy M, Wilson BC. Apoptosis induced in vivo by photodynamic therapy in normal brain and intracranial tumour tissue. British journal of cancer. 2000;83(8):1110-7. 
17. Bisland SK, Lilge L, Lin A, Rusnov R, Wilson BC. Metronomic photodynamic therapy as a new paradigm for photodynamic therapy: rationale and preclinical evaluation of technical feasibility for treating malignant brain tumors. Photochemistry and photobiology. 2004;80:22-30.

18. Chopp M, Madigan L, Dereski M, Jiang F, Li Y. Photodynamic therapy of human glioma (U87) in the nude rat. Photochemistry and photobiology. 1996;64(4):707-11.

19. Tetard MC, Vermandel M, Leroy HA, Leroux B, Maurage CA, Lejeune JP, et al. Interstitial 5-ALA photodynamic therapy and glioblastoma: Preclinical model development and preliminary results. Photodiagnosis Photodyn Ther. 2016;13:218-24.

20. Olzowy B, Hundt CS, Stocker S, Bise K, Reulen HJ, Stummer W. Photoirradiation therapy of experimental malignant glioma with 5-aminolevulinic acid. J Neurosurg. 2002;97(4):970-6.

21. Schuierer G, Reimer P, Allkemper T, Peters PE. [Fast and ultra-fast MRI of the brain]. Radiologe. 1995;35(12):894-901.

22. Wong EC, Buxton RB, Frank LR. Implementation of quantitative perfusion imaging techniques for functional brain mapping using pulsed arterial spin labeling. NMR in biomedicine. 1997;10(4-5):237-49.

23. Teng L, Nakada M, Zhao SG, Endo Y, Furuyama N, Nambu E, et al. Silencing of ferrochelatase enhances 5-aminolevulinic acid-based fluorescence and photodynamic therapy efficacy. Br J Cancer. 2011;104(5):798-807.

24. Dupont C, Vermandel M, Leroy HA, Quidet M, Lecomte F, Delhem N, et al. INtraoperative photoDYnamic Therapy for GliOblastomas: Study Protocol for a Phase I Clinical Trial. Neurosurgery. 2018. 